\title{
ALBANESE MAPS AND FUNDAMENTAL GROUPS OF VARIETIES WITH MANY RATIONAL POINTS OVER FUNCTION FIELDS
}

\author{
ARIYAN JAVANPEYKAR AND ERWAN ROUSSEAU
}

\begin{abstract}
We investigate properties of the Albanese map and the fundamental group of a complex projective variety with many rational points over some function field, and prove that every linear quotient of the fundamental group of such a variety is virtually abelian, as well as that its Albanese map is surjective, has connected fibres, and has no multiple fibres in codimension one.
\end{abstract}

\section{INTRODUCTION}

The abundance of rational points on a variety over a number field is conjectured to force the fundamental group of the underlying topological space to exhibit various rigidity properties. Indeed, it seems reasonable to suspect that the topological fundamental group of a smooth projective variety over a number field with a dense set of rational points contains a finite index abelian subgroup. In this paper we verify such expectations for varieties with a dense set of rational points over a function field of characteristic zero.

1.1. Lang's conjectures. Let $k$ be an algebraically closed field of characteristic zero. Lang conjectured that varieties of general type over $k$ satisfy certain finiteness properties (see Lan86 and [Jav §12]), where we say that a proper (integral) variety $X$ over $k$ is of general type if for some (hence any) proper desingularization $\widetilde{X} \rightarrow X$ we have that $\omega_{\widetilde{X}}$ is big. To state the relevant version of Lang's conjecture, we follow [BJK, Jav] and introduce the class of pseudo-geometrically hyperbolic varieties.

Definition 1.1 (Pseudo-geometric hyperbolicity). A variety $X$ over $k$ is pseudo-geometrically hyperbolic over $k$ if there is a proper closed subset $Z \subsetneq X$ such that, for every $x$ in $X(k) \backslash Z$ and every smooth connected pointed curve $(C, c)$ over $k$, the set $\operatorname{Hom}_{k}((C, c),(X, x))$ of morphisms $f:(C, c) \rightarrow(X, x)$ is finite.

We will also say that a variety $X$ over $k$ is geometrically hyperbolic over $k$ if one can take $Z=\emptyset$ in the above definition i.e., for every $x$ in $X(k)$ and every smooth connected pointed curve $(C, c)$ over $k$, the set $\operatorname{Hom}_{k}((C, c),(X, x))$ is finite. Note that "being geometrically hyperbolic over $k$ " means, roughly speaking, that, for any smooth connected curve $C$ with function field $K$, the variety $X_{k(t)}$ over the function field $k(t)$ has only finitely many "pointed" $K$-rational points. Similarly, "being pseudo-geometrically hyperbolic" is similar to having such a finiteness property for "pointed" rational points outside some exceptional locus $Z$.

The starting point of our paper is the following finiteness conjecture for varieties.

Conjecture 1.2 (Consequence of Lang's conjectures). A projective (integral) variety $X$ over $k$ is of general type if and only if it is pseudo-geometrically hyperbolic over $k$.

For the reader's convenience we briefly explain how Conjecture 1.2 is related to Lang's conjectures, as this might be unclear to non-experts. First, Lang's conjectures predict that a projective variety of general type is pseudo-algebraically hyperbolic (Definition 2.4), and a simple application of Mori's bend-and-break shows that a pseudo-algebraically hyperbolic scheme is pseudo-geometrically hyperbolic (see Proposition 2.5). On the other hand, a pseudo-algebraically hyperbolic variety is pseudo-groupless [JX, Proposition 5.4], and Lang conjectured that pseudo-groupless projective varieties are of general type. In fact, the latter conjecture also follows from combining the abundance conjecture in the MMP with "standard" conjectures

2010 Mathematics Subject Classification. 32Q45, 32M15, 37F75.

Key words and phrases. Hyperbolicity, Lang's conjectures, Campana's conjectures, special varieties, rational points, function fields, Albanese variety, fundamental groups, period maps, Shafarevich maps, orbifold pairs.

The second author was supported by the ANR project "FOLIAGE", ANR-16-CE40-0008 and by the European Union's Horizon 2020 research and innovation program under the Marie Sklodowska-Curie grant agreement No 75434. 
on rational curves on Calabi-Yau varieties. For example, note that groupless surfaces are of general type (see [JX, Lemma 3.23]).

The above consequence of Lang's conjecture (which we simply refer to as Lang's conjecture) is known in dimension one, but open in dimension two. We do note that every proper pseudo-geometrically hyperbolic surface is of general type (see [Jav, Proposition 11.7] for example); such a statement is not known currently in dimension three. Moreover, as we show in Section 3.3 Lang's conjecture holds for normal projective varieties whose Albanese map is generically finite onto its image. Finally, for other non-trivial examples for which we know Lang's conjecture to hold we refer the reader to Section 2.1

Lang's conjecture is concerned with the finiteness of rational points on varieties over function fields. We will however be interested in varieties with a lot of rational points over function fields, i.e., those that behave "opposite" to pseudo-geometrically hyperbolic varieties. This brings us to Campana's conjectures on special varieties.

1.2. Campana's conjectures. Campana's conjectures provide a picture complementary to that of Lang's conjectures on rational points and entire curves on varieties of general type. To explain this, for $X$ a proper variety over $k$, we follow Campana and say that $X$ is special (over $k$ ) if, for some (hence every) desingularization $\widetilde{X} \rightarrow X$, for every integer $p \geq 1$ and every rank one coherent subsheaf $F \subset \Lambda^{p} \Omega_{\widetilde{X}}^{1}$, the Kodaira dimension of $F$ is at most $p-1$; see Cam04, Cam11. Roughly speaking, a variety is special if it is as far away as possible from being of general type. Just like being of general type, this notion is insensitive to field extensions, i.e., if $L / k$ is an extension of algebraically closed fields of characteristic zero and $X$ is a proper variety over $k$, then $X$ is special over $k$ if and only if $X_{L}$ is special over $L$ (see Cam04, Proposition 9.18]).

Before we state the version of Campana's conjecture we are interested in, we recall his analytic and arithmetic conjectures.

Definition 1.3. A variety $X$ over $\mathbb{C}$ is Brody-special if there is a (Zariski-)dense holomorphic map $\mathbb{C} \rightarrow$ $X^{\text {an }}$.

Conjecture 1.4 (Campana's analytic conjecture). A projective variety $X$ over $\mathbb{C}$ is special if and only if it is Brody-special.

A non-constant holomorphic map $\mathbb{C} \rightarrow X^{\text {an }}$ is usually referred to as an entire curve in $X$, and a variety $X$ over $\mathbb{C}$ is said to be pseudo-Brody hyperbolic if there is a proper (Zariski-)closed subset $Z \subsetneq X$ such that every entire curve $\mathbb{C} \rightarrow X^{\text {an }}$ factors over $Z^{\text {an }}$. Just as positive-dimensional special varieties are as far away as possible from being of general type, a positive-dimensional Brody-special variety is as far away as possible from being pseudo-Brody hyperbolic. Therefore, Campana's analytic conjecture provides a complementary picture to the Green-Griffiths-Lang conjecture that a proper variety is of general type if and only if it is pseudo-Brody hyperbolic. (To be precise, Green-Griffiths and Lang explicitly conjectured that a projective variety of general type is pseudo-Brody hyperbolic. The converse statement is implicit in Lang's paper; see [Lan86, p. 161].)

Intuitively speaking, the existence of a dense entire curve on a positive-dimensional variety over a number field should be equivalent to the existence of many rational points. To make this more precise, we extend Campana's notion of arithmetically-special variety to varieties over arbitrary algebraically closed fields of characteristic zero.

Definition 1.5. A proper variety $X$ over $k$ is arithmetically-special over $k$ if there exists a subfield $K \subset k$ which is a finitely generated field extension of $\mathbb{Q}$ and a model $\mathcal{X}$ for $X$ over $K$ such that $\mathcal{X}(K)$ is dense in $X$.

The notion of "arithmetic-specialness" introduced here is slightly more general than that of Campana who restricts himself to varieties over $\overline{\mathbb{Q}}$. (Note that a variety over $\overline{\mathbb{Q}}$ is arithmetically-special in the above sense if and only if there is a number field $K \subset \overline{\mathbb{Q}}$ and a model $\mathcal{X}$ for $X$ over $K$ such that $\mathcal{X}(K)$ is dense.)

The arithmetic analogue of Conjecture 1.4 then reads as follows.

Conjecture 1.6 (Campana's arithmetic conjecture). A projective variety $X$ over $k$ is special over $k$ if and only if it is arithmetically-special over $k$. 
A proper variety $X$ over $k$ is pseudo-Mordellic over $k$ if there is a proper closed subset $Z \subsetneq k$ such that, for every subfield $K \subset k$ which is a finitely generated field extension of $\mathbb{Q}$ and every model $\mathcal{X}$ for $X$ over $K$, the set $\mathcal{X}(K) \backslash Z$ is finite. Just as positive-dimensional special varieties are as far away as possible from being of general type, a positive-dimensional arithmetically-special variety is as far away as possible from being pseudo-Mordellic. Therefore, Campana's conjecture complements Lang's conjecture that a projective variety $X$ over $k$ is of general type if and only if $X$ is pseudo-Mordellic over $k$.

Inspired by Campana's analytic and arithmetic conjectures, and led by the analogy between function fields and number fields (see for instance [Jav Remark 11.3]), we introduce a "special" counterpart to the notion of pseudo-geometric hyperbolicity (Definition 1.1).

Definition 1.7 (Geometrically special varieties). A variety $X$ over $k$ is geometrically-special over $k$ if, for every dense open subset $U \subset X$, there exists a smooth quasi-projective connected curve $C$ over $k$, a point $c$ in $C(k)$, a point $u$ in $U(k)$, and a sequence of morphisms $f_{i}: C \rightarrow X$ with $f_{i}(c)=u$ for $i=1,2, \ldots$ such that $C \times X$ is covered by the graphs $\Gamma_{f_{i}} \subset C \times X$ of these maps, i.e., the closure of $\cup_{i=1}^{\infty} \Gamma_{f_{i}}$ equals $C \times X$.

Note that the density of $\cup_{i=1}^{\infty} \Gamma_{f_{i}}$ in this definition is equivalent to the density of the $K(C)$-rational points of $X_{K(C)}$ induced by morphisms $C \rightarrow C \times X$ with $f(c)=(c, x)$. That is, roughly speaking, a variety is geometrically-special if and only if the set of "pointed" rational points of $X_{k(t)}$ is potentially dense. Thus, intuitively speaking, to be geometrically-special is to admit an abundant set of rational points over some function field.

Just as positive-dimensional special varieties are as far away from being of general type, a positivedimensional geometrically-special is as far away as possible from being pseudo-geometrically hyperbolic. For example, a geometrically-special variety does not dominate a positive-dimensional pseudo-geometrically hyperbolic variety (see Corollary 2.9).

Following Campana's philosophy on special varieties, we expect that geometric-specialness coincides with being special.

Conjecture 1.8 (Inspired by Campana). A projective variety $X$ over $k$ is special over $k$ if and only if it is geometrically-special over $k$.

The aim of this paper is to provide evidence for Conjecture 1.8 We stress that this conjecture fits in well with the parellelism between Campana's and Lang's conjecture, as illustrated by the following table.

\begin{tabular}{|l|l|}
\hline \multicolumn{2}{|c|}{ Lang and Campana conjectures } \\
\hline Notion of pseudo-hyperbolicity & Notion of specialness \\
\hline General type & Special \\
Pseudo-Brody-hyperbolic & Brody-special \\
Pseudo-Mordellic & Arithmetically-special \\
Pseudo-geometrically-hyperbolic & Geometrically-special. \\
\hline
\end{tabular}

Lang's conjecture says that the four notions on the left hand side are equivalent for any projective variety $X$ over $k$, whereas Campana's conjecture and Conjecture 1.8 predict that the four notions on the right hand side are equivalent. Except for some particular cases (e.g,. $X$ a closed subvariety of an abelian variety) both conjectures are still wide open.

1.3. The Albanese map of a special variety. We will use the following terminology.

Definition 1.9. A surjective morphism $f: X \rightarrow Y$ of varieties over $k$ is said to have no multiple fibres in codimension one if, for every point $y$ in $Y$ of codimension one, the scheme-theoretic fibre $X_{y}$ has an irreducible component which is generically reduced. More concretely, if $f: X \rightarrow Y$ has no multiple fibres in codimension one and we write $X_{y}=\sum_{F} n_{F} F$, where the sum runs over all irreducible components of $X_{y}$ and $n_{F}$ is the multiplicity of $F$ in $X_{y}$ (see [Sta15, Tag 02QU]), then there is a component $F$ of $X_{y}$ such that $n_{F}=1$.

Our first main result concerns the structure of the Albanese map of a geometrically-special normal projective variety. We recall that, given a normal proper (integral) variety over $k$, the Albanese variety of $X$ is $\operatorname{Alb}(X):=\left(\mathrm{Pic}_{X / k}^{0}\right)_{\text {red}}$, and we refer the reader to [Moc12, Proposition A.6] for basic properties of the Albanese map. (Strictly speaking, the Albanese map $X \rightarrow \operatorname{Alb}(X)$ is associated to the choice of a point $x$ in $X(k)$. However, the properties we are interested in are independent of the choice of $x$ in $X(k)$.) 
ARIYAN JAVANPEYKAR AND ERWAN ROUSSEAU

Theorem 1.10 (Main Result, I). If $X$ is a normal projective geometrically-special variety over $k$, then the Albanese map $X \rightarrow \mathrm{Alb}(X)$ is surjective, has connected fibres, and has no multiple fibers in codimension one.

Our proof of Theorem 1.10 relies on Yamanoi's seminal work on holomorphic curves in algebraic varieties of maximal Albanese dimension Yam15. In particular, although the statements are purely algebraic, we implicitly rely on Yamanoi's work on (complex-analytic) Nevanlinna theory.

Theorem 1.10 fits in perfectly with Conjecture 1.8 as the following result of Campana (see Cam04, Proposition 5.3]) shows.

Theorem 1.11 (Campana). If $X$ is a normal projective special variety over $k$, then the Albanese map $X \rightarrow \operatorname{Alb}(X)$ is surjective, has connected fibres, and has no multiple fibres in codimension one.

We mention that, as explained in [CC16, Proposition 5.1], for $X$ a smooth projective Brody-special variety over $\mathbb{C}$, the Albanese map $X \rightarrow \operatorname{Alb}(X)$ is surjective, has connected fibres, and has no multiple fibres in codimension one (see also Remark 3.27). On the other hand, for $X$ a smooth projective arithmeticallyspecial variety over $k$, the Albanese map $X \rightarrow \operatorname{Alb}(X)$ is surjective by Faltings's theorem Fal94. In light of Campana's conjectures, the fibers of the Albanese map should even be connected. However, as we do not have at our disposal any satisfying results on the finiteness of rational points for ramified covers of abelian varieties (see $\mathrm{CDJ}^{+}$for recent progress nonetheless), proving this connectedness seems out of reach at the moment.

1.4. The abelianity conjecture. For $X$ a connected variety over $\mathbb{C}$, we let $\pi_{1}(X)$ be the (topological) fundamental group of $X^{\text {an }}$ (with respect to the choice of some basepoint). Note that the étale fundamental group $\pi_{1}^{\text {ét }}(X)$ of $X$ is the profinite completion of $\pi_{1}(X)$.

Campana conjectured that the fundamental group $\pi_{1}(X)$ of a smooth projective connected special variety over $\mathbb{C}$ is virtually abelian (i.e., contains a finite index abelian subgroup).

Conjecture 1.12 (Campana's abelianity conjecture). Let $X$ be a smooth projective connected variety over $\mathbb{C}$. Then the following statements hold.

(1) If $X$ is special, then $\pi_{1}(X)$ is virtually abelian.

(2) If $X$ is Brody-special, then $\pi_{1}(X)$ is virtually abelian.

(3) If $X$ is arithmetically-special over $\mathbb{C}$, then $\pi_{1}(X)$ is virtually abelian.

(4) If $X$ is geometrically-special over $\mathbb{C}$, then $\pi_{1}(X)$ is virtually abelian.

This conjecture is still open. However, under the additional assumption that $\pi_{1}(X)$ admits a faithful linear complex representation, this conjecture is resolved in cases (1) and (2) by Campana and Yamanoi, respectively. Their precise results are as follows (see Cam04, Theorem 7.8] and Yam10, Theorem 1.1]).

Theorem 1.13 (Campana). If $X$ is a smooth projective special variety over $\mathbb{C}$, then the image of any homomorphism $\pi_{1}(X) \rightarrow \mathrm{GL}_{n}(\mathbb{C})$ is virtually abelian.

Theorem 1.14 (Yamanoi). If $X$ is a smooth projective Brody-special variety over $\mathbb{C}$, then the image of any homomorphism $\pi_{1}(X) \rightarrow \mathrm{GL}_{n}(\mathbb{C})$ is virtually abelian.

Motivated by Conjecture [1.8. Conjecture 1.12, Campana's theorem (Theorem 1.13), and Yamanoi's theorem (Theorem 1.14), we prove the following result.

Theorem 1.15 (Main Result, II). If $X$ is a smooth projective geometrically-special variety over $\mathbb{C}$, then the image of any homomorphism $\pi_{1}(X) \rightarrow \mathrm{GL}_{n}(\mathbb{C})$ is virtually abelian.

Our final result pushes Theorem 1.15 a bit further. To state it, we follow [Yam10, p. 552] and recall that a representation $\rho: \pi_{1}(X) \rightarrow \mathrm{GL}_{n}(\mathbb{C})$ is big if there is a countable collection of proper closed subsets $X_{i} \subsetneq X$ with $i=1, \ldots$ such that, for every positive-dimensional subvariety $Z \subset X$ containing a point of $X \backslash \cup_{i=1}^{\infty} X_{i}$, the group $\rho\left[\operatorname{Im}\left(\pi_{1}(\tilde{Z}) \rightarrow \pi_{1}(X)\right)\right]$ is infinite, where $\tilde{Z} \rightarrow Z$ is a desingularization of $Z$.

Theorem 1.16 (Main Result, III). Let $X$ be a smooth projective connected variety over $\mathbb{C}$ with a big representation $\rho: \pi_{1}(X) \rightarrow \mathrm{GL}_{n}(\mathbb{C})$. If $X$ is geometrically-special, then there exists a finite étale covering $Y \rightarrow X$ which is birationally equivalent to an abelian variety. 
The proofs of Theorem 1.15and1.16 rely on our structure result for the Albanese map of a geometricallyspecial variety (Theorem 1.10), foundational results from Hodge theory due to Deligne, Griffiths, and Schmid (see Lemma 4.3), as well as the recent resolution of Griffiths's algebraicity conjectures for period maps by Bakker-Brunebarbe-Tsimerman (see Theorem 4.4), Zuo's results on spectral covers (see the proof of Theorem 4.1), and the theory of Shafarevich maps (see the proof of Proposition 4.2). Arguably, the novel technical result in our proof of Theorem 1.15 is Theorem 4.1 .

1.5. Outline of paper. In Section 2 we prove basic properties of geometrically-special varieties such as the invariance by finite étale covers (Lemma 2.11), as well as the fact that the image of a geometrically-special variety is geometrically-special (Lemma 2.8). In Section 3 we show that abelian varieties are geometricallyspecial (Proposition 3.1), and prove the structure result for the Albanese map of a geometrically-special projective variety (Theorem 1.10) by applying Yamanoi's results on the pseudo-hyperbolicity of ramified covers of abelian varieties, as well as Yamanoi's work on the pseudo-hyperbolicity of orbifold pairs on abelian varieties (see Section 3.6). In Section 4, we prove that linear quotients of the fundamental group of a smooth projective connected geometrically-special variety are virtually abelian.

Acknowledgements. The ideas of Campana and Yamanoi have had an enormous impact on this paper. We are grateful to both of them for their past work without which this paper would not have existed. We thank Philipp Habegger and Junyi Xie for telling us about Lemma 2.17. The first named author thanks the IHES and the University of Paris-Saclay for their hospitality. Part of this work was done during the visit of the first and second named author at the Freiburg Institute for Advanced Studies; they thank the Institute for providing an excellent working environment. We are grateful to the referees for many useful comments.

Conventions. Throughout this paper, we let $k$ be an algebraically closed field of characteristic zero. A variety over $k$ is a finite type separated integral (i.e., irreducible and reduced) scheme over $k$.

If $X$ is a variety over $k$ and $A \subset k$ is a subring, then a model for $X$ over $A$ is a pair $(\mathcal{X}, \phi)$ with $\mathcal{X} \rightarrow \operatorname{Spec} A$ a finite type separated scheme and $\phi: \mathcal{X} \otimes_{A} k \rightarrow X$ an isomorphism of schemes over $k$. We omit $\phi$ from our notation.

A pointed variety (over $k$ ) is a pair $(V, v)$, where $V$ is a variety over $k$ and $v$ is an element of $V(k)$. A pointed variety $(C, c)$ is a pointed curve if $C$ is pure of dimension one. We say that a pointed variety $(V, v)$ is smooth (resp. connected, resp. projective) if $V$ is smooth (resp. connected, resp. projective). A morphism $(V, v) \rightarrow\left(V^{\prime}, v^{\prime}\right)$ of pointed varieties consists of the data of a morphism $f: V \rightarrow V^{\prime}$ of varieties over $k$ such that $f(v)=v^{\prime}$.

If $X$ is a locally finite type scheme over $\mathbb{C}$, we let $X^{\text {an }}$ be the associated complex-analytic space.

\section{Geometrically-Special varieties}

Guided by the expectation that geometric-specialness coincides with Campana's notion of specialness (Conjecture 1.8), we will prove several basic properties of geometrically-special varieties in this section; none of the results in this section are particularly hard to prove.

Let $k$ be an algebraically closed field of characteristic zero. Recall that a variety $X$ over $k$ is geometricallyspecial over $k$ if, for every dense open subset $U \subset X$, there exists a smooth affine connected pointed curve $(C, c)$, a point $x$ in $U(k)$, and a sequence of morphisms $\left\{f_{i}:(C, c) \rightarrow(X, u)\right\}_{i=1}^{\infty}$ such that the closure of $\cup_{i=1}^{\infty} \Gamma_{f_{i}}$ in $C \times X$ equals $C \times X$ (see Definition [1.7). We will sometimes refer to a sequence of morphisms $f_{i}:(C, c) \rightarrow(X, u)$ such that the closure of $\cup_{i=1}^{\infty} \Gamma_{f_{i}}$ equals $C \times X$ as a covering set for $X$ (even though it is really a "covering" of $C \times X)$.

Clearly, a proper variety $X$ over $k$ is geometrically-special over $k$ if and only if, for every dense open subset $U \subset X$, there exists a smooth projective connected pointed curve $(C, c)$ over $k$, a point $u$ in $U(k)$, and a covering set $f_{i}:(C, c) \rightarrow(X, u)$ for $X$. The existence of a covering set here is in fact equivalent to the the universal evaluation map

$$
C \times \underline{\operatorname{Hom}}_{k}((C, c),(X, x)) \rightarrow C \times X
$$

being dominant, where $\underline{\operatorname{Hom}}_{k}((C, c),(X, x))$ denotes the moduli scheme of morphisms $(C, c) \rightarrow(X, x)$ over $k$; this moduli scheme is a countable union of quasi-projective varieties over $k$. 
Being geometrically-special is related to potential density of "pointed" rational points, as we briefly explain in the following remark.

Remark 2.1. Let $K$ be the function field of a smooth connected curve $C$ over $k$, and let $X$ be a variety over $\bar{K}$. We say that $X$ satisfies $k$-pointed-potential density if, for every dense open subset $U \subset X$, there is a point $u$ in $U$, a curve $D$ over $k$ quasi-finite over $C$, a point $d$ in $D(k)$, and a model $(\mathcal{X}, x)$ for $(X, x)$ over $D$ such that

$$
\operatorname{Im}\left[\operatorname{Hom}_{C}((D, d),(\mathcal{X}, x)) \rightarrow X(\bar{K})\right]
$$

is dense in $X$. This extends the above definition in the following sense. A variety $X$ over $k$ is geometricallyspecial over $k$ if and only if (the "constant" variety) $X_{\overline{k(t)}}$ satisfies $k$-pointed-potential density.

Note that Conjecture 1.8 is concerned with isotrivial varieties over $k(t)$. On the other hand, even in the non-isotrivial case, it seems reasonable to supect density of "pointed rational points"; a similar conjecture was stated for (non-pointed) rational points on non-isotrivial varieties by Campana [Cam11.

2.1. Algebraic and geometric hyperbolicity. To keep track of the "exceptional locus" of a pseudogeometrically hyperbolic variety (Definition 2.2 ), we use the notion of geometric hyperbolicity modulo a closed subset.

Definition 2.2. Let $X$ be a variety over $k$. If $Z$ is a closed subset of $X$, then $X$ is geometrically hyperbolic modulo $Z$ if, for every $x$ in $X(k) \backslash Z$ and every smooth connected pointed curve $(C, c)$ over $k$, the set $\operatorname{Hom}_{k}((C, c),(X, x))$ is finite.

Bounding the degrees of maps from curves into a compact space forces finiteness properties of sets of pointed maps (see JK20 for precise statements). To make this more precise, we recall Demailly's notion of algebraic hyperbolicity [Dem97]; we discuss this notion further in the orbifold setting in Section 3.5.

Definition 2.3. Let $X$ be a projective variety and let $E \subset X$ be a closed subset. Then $X$ is algebraically hyperbolic modulo $E$ over $k$ if, for every ample line bundle $L$ on $X$, there is a constant $\alpha_{X, L, E}$ such that, for every smooth projective connected curve $C$ over $k$, and every morphism $f: C \rightarrow X$ with $f(C) \not \subset E$, the inequality

$$
\operatorname{deg}_{C} f^{*} L \leq \alpha_{X, L, E} \cdot \operatorname{genus}(C)
$$

holds.

Definition 2.4. A projective variety $X$ is pseudo-algebraically hyperbolic over $k$ if there is a proper closed subset $E \subsetneq X$ such that $X$ is algebraically hyperbolic modulo $E$.

We will use the following simple application of Mori's bend-and-break (see [JX], Theorem 1.12.(2)]).

Proposition 2.5. If $X$ is a projective pseudo-algebraically hyperbolic scheme over $k$, then $X$ is pseudogeometrically hyperbolic over $k$.

Since a positive-dimensional projective geometrically-special scheme over $k$ is not pseudo-geometrically hyperbolic, it follows from Proposition 2.5 that such a variety is also not pseudo-algebraically hyperbolic.

For the reader's convenience, we give several examples of pseudo-geometrically hyperbolic varieties. For example, if $X$ is a proper Brody hyperbolic variety over $\mathbb{C}$, then $X$ is geometrically hyperbolic over $\mathbb{C}$ (see [Kob98, Theorem 5.3.10]), and even algebraically hyperbolic [Dem97. In the non-compact case, if $X$ is a smooth affine variety which admits a hyperbolic embedding [Kob98, Chapter 3.3], then $X$ is geometrically hyperbolic (see [JLa, Theorem 1.7]). Furthermore, if $X$ is a smooth projective connected surface of general type with $c_{1}^{2}>c_{2}$, then the projective surface $X$ is pseudo-algebraically hyperbolic (and thus pseudogeometrically hyperbolic) by Bogomolov's theorem Bog77]. Finally, examples of geometrically hyperbolic moduli spaces of polarized varieties are given in JLb, JSZ.

2.2. Birational invariance. The notions of being special, Brody-special, and arithmetically-special are "birational". We now show that the same holds for being geometrically-special (see Lemma 2.8).

Lemma 2.6 (Going up). Let $X \rightarrow Y$ be a proper birational morphism of varieties over $k$. If $Y$ is geometrically-special over $k$, then $X$ is geometrically-special. 
Proof. Let $y \in Y$ be a point not contained in the exceptional locus of $X \rightarrow Y$ for which we can choose a pointed curve $(C, c)$ and a covering set $f_{i}:(C, c) \rightarrow(Y, y)$. Let $x \in X$ be the unique point of $X$ lying over $y$. Then, as $y$ is not contained in the exceptional locus of the proper morphism $X \rightarrow Y$, we have that each $f_{i}$ lifts uniquely to a map $g_{i}:(C, c) \rightarrow(X, x)$. It is clear that these maps form a covering set for $(X, x)$, i.e., the union of the graphs $\Gamma_{g_{i}}$ in $C \times X$ is dense in $X$. Clearly, this implies that $X$ is geometrically-special over $k$.

Lemma 2.7 (Going down). Let $f: X \rightarrow Y$ be a surjective morphism of varieties over $k$. If $X$ is geometrically-special over $k$, then $Y$ is geometrically-special over $k$.

Proof. Let $V \subset Y$ be a dense open subset, and let $U:=f^{-1}(V)$. Since $X$ is geometrically-special over $k$, there is a pointed curve $(C, c)$ over $k$, a point $u$ in $U(k)$, and a covering set $f_{i}:(C, c) \rightarrow(X, x)$. Define $v:=f(u)$, and $g_{i}:=f \circ f_{i}$. Then $g_{i}(c)=v$ and $C \times Y$ is covered by the graphs of the maps $g_{i}$, so that the $g_{i}:(C, c) \rightarrow(Y, v)$ form a covering set for $Y$. We conclude that $Y$ is geometrically-special over $k$, as required.

Lemma 2.8. Let $X \rightarrow Y$ be a dominant rational map of proper varieties over $k$. Then the following statements hold.

(1) If $X$ is special, then $Y$ is special.

(2) Assume $k=\mathbb{C}$. If $X$ is Brody-special, then $Y$ is Brody-special.

(3) If $X$ is arithmetically-special over $k$, then $Y$ is arithmetically-special over $k$.

(4) If $X$ is geometrically-special over $k$, then $Y$ is geometrically-special over $k$.

Proof. Note that (1) is due to Campana [Cam04, and that (2) and (3) are obvious. To prove (4), let $Z \rightarrow X$ be a proper birational surjective morphism such that the composed rational map $Z \rightarrow X \rightarrow Y$ is a (proper surjective) morphism. First, as $X$ is geometrically-special over $k$, it follows that $Z$ is geometrically-special (Lemma 2.6). Then, as $Z$ is geometrically-special over $k$ and $Z \rightarrow Y$ is surjective, we conclude that $Y$ is geometrically-special over $k$ (Lemma 2.7).

Corollary 2.9. Let $X \rightarrow Y$ be a dominant rational map of proper varieties. If $\operatorname{dim} Y \geq 1$ and $X$ is geometrically-special over $k$, then $Y$ is not pseudo-geometrically hyperbolic over $k$.

Proof. By Lemma 2.8. (4) we have that $Y$ is geometrically-special. This proves the corollary, as a positivedimensional geometrically-special variety is not pseudo-geometrically hyperbolic.

2.3. Finite étale maps. The fact that specialness is preserved under taking finite étale covers was proven by Campana. The corresponding result for geometrically-special varieties requires a well-known finiteness result for finite étale covers of varieties. Note that, in this paper, the following finiteness result is only used in the case that $X$ is a curve (in which case it is easier to prove); we include the more general statement for the sake of future reference.

Lemma 2.10 (Finiteness). Let $d \geq 1$ be an integer, and let $X$ be a variety over $k$. Then, the set of $X$-isomorphism classes of finite étale morphisms $Y \rightarrow X$ of degree $d$ is finite.

Proof. We may and do assume that $k=\mathbb{C}$. Then, the result follows from the fact that the (topological) fundamental group $\pi_{1}(X)$ of $X$ is finitely generated (see Gro72, Exposé II, Théorème 2.3.1]).

Lemma 2.11. Let $\pi: X \rightarrow Y$ be a finite étale morphism of varieties over $k$. Then $X$ is geometricallyspecial over $k$ if and only if $Y$ is geometrically-special over $k$.

Proof. Assume that $X$ is geometrically-special over $k$. Since $\pi: X \rightarrow Y$ is surjective, it follows from Lemma 2.8 that $Y$ is geometrically-special over $k$.

Conversely, assume that $Y$ is geometrically-special over $k$. Let $U \subset X$ be a dense open subset such that $\left.\pi\right|_{U}: U \rightarrow \pi(U)$ is finite étale of degree $\operatorname{deg} \pi$. Let $y$ be a point in the open subset $\pi(U)$ of $Y$ for which we can choose a covering set $f_{i}:(C, c) \rightarrow(Y, y)$ with $i=1,2, \ldots$. For each $i$, consider the Cartesian diagram

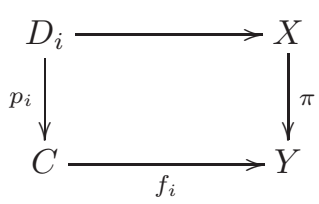


Since $p_{i}$ is a finite étale (surjective) morphism of degree at most $\operatorname{deg}(\pi)$, the set of isomorphism classes of the curves $D_{i}$ is finite (Lemma 2.10). Thus, replacing the $f_{i}$ by a suitable subsequence if necessary, we see that there is a point $x$ in $\pi^{-1}\{y\}$, a smooth connected pointed curve $(D, d)$ over $k$, and a covering set $g_{i}:(D, d) \rightarrow(X, x)$.

Now, to show that $X$ is geometrically-special over $k$, let $U \subset X$ be a dense open. Choose a dense open $U^{\prime} \subset U$ such that $\pi_{U^{\prime}}: U^{\prime} \rightarrow \pi\left(U^{\prime}\right)$ is finite étale of degree $\operatorname{deg} \pi$. Then, we have shown that there is a point $x$ in $U^{\prime} \subset U$, a smooth connected pointed curve $(D, d)$ over $k$, and a covering set $g_{i}:(D, d) \rightarrow(X, x)$. This concludes the proof.

Lemma 2.11 fits in well with Campana's conjectures, as the following proposition shows (included for the sake of completeness).

Proposition 2.12. Let $Y \rightarrow X$ be a finite étale morphism of proper varieties over $k$. Then the following statements hold.

(1) The variety $Y$ is special over $k$ if and only if $X$ is special over $k$.

(2) If $k=\mathbb{C}$, then $Y$ is Brody-special if and only if $X$ is Brody-special.

(3) $Y$ is arithmetically-special over $k$ if and only if $X$ is arithmetically-special over $k$.

(4) $Y$ is geometrically-special over $k$ if and only if $X$ is geometrically-special over $k$.

Proof. Note that (1) follows from [Cam04, Theorem 5.12], and that (2) is obvious. Moreover, a standard "Chevalley-Weil" type argument shows that $X$ is arithmetically-special over $k$ if and only if $Y$ is arithmetically-special over $k$ (see for example [JLb, Lemma 8.2]). This proves (3). Finally, (4) follows from (the more general) Lemma 2.11.

2.4. Products. It is not hard to see that if $X$ and $Y$ are arithmetically-special over $k$, then $X \times Y$ is arithmetically-special over $k$. Moreover, if $k=\mathbb{C}$ and $X$ and $Y$ both admit a dense entire curve, say $f: \mathbb{C} \rightarrow X^{\text {an }}$ and $g: \mathbb{C} \rightarrow X^{\text {an }}$, then $(f, g): \mathbb{C}^{2} \rightarrow X \times Y$ has Zariski dense image. Thus, if $\mathbb{C} \rightarrow \mathbb{C}^{2}$ is a metrically-dense entire curve, then the composed map $\mathbb{C} \subset \mathbb{C}^{2} \rightarrow X \times Y$ is a dense entire curve. Therefore, if $X$ and $Y$ are Brody-special, then $X \times Y$ is Brody-special. Finally, if $X$ and $Y$ are smooth projective special varieties over $k$, then $X \times Y$ is special by a theorem of Campana Cam04. This "product property" also holds for geometrically-special varieties.

Lemma 2.13. Let $X$ and $Y$ be geometrically-special varieties over $k$. Then $X \times Y$ is a geometrically-special variety over $k$.

Proof. Let $g_{i}:\left(C_{1}, c_{1}\right) \rightarrow(X, x)$ and $h_{i}:\left(C_{2}, c_{2}\right) \rightarrow(Y, y)$ be a covering set for $X$ and $Y$, respectively. If $i \geq 1$ and $j \geq 1$, consider

$$
f_{i, j}:\left(C_{1} \times C_{2},\left(c_{c}^{\prime}\right)\right) \rightarrow(X \times Y,(x, y)), \quad f_{i, j}(a, b)=\left(g_{i}(a), h_{j}(b)\right) .
$$

Note that the (countable) union of the graphs $\cup_{i, j} \Gamma_{f_{i, j}}$ of the morphisms $f_{i, j}$ is dense in $C_{1} \times C_{2} \times X$. Let $C \subset C_{1} \times C_{2}$ be a smooth connected very ample divisor containing $\left(c, c^{\prime}\right)$. Then each morphism $\left.f_{i, j}\right|_{C}: C \rightarrow X \times Y$ maps $\left(c, c^{\prime}\right)$ to $(x, y)$, and the union of their graphs is dense in $C \times X$. This shows that $X \times Y$ is geometrically-special over $k$.

2.5. Rationally connected varieties. A smooth projective variety over $k$ is rationally connected if any two general points $p, q$ on $X$ can be connected by a chain of rational curves (see [Deb01, Definition 4.3]) Campana showed that such a variety is special (see Cam04]), and a recent theorem of CampanaWinkelmann shows that a rationally connected smooth projective variety is Brody-special. It is straightforward to verify that such a variety is geometrically-special, as we show now.

Proposition 2.14. If $X$ is a smooth projective rationally connected variety over $k$, then $X$ is geometricallyspecial over $k$.

Proof. Note that, there is a dense open subset $U \subset X$ such that, for every $x$ in $U(k)$, the image of the evaluation morphism

$$
\mathrm{ev}_{\infty}: \underline{\operatorname{Hom}}\left(\left(\mathbb{P}_{k}^{1}, 0\right),(X, x)\right) \rightarrow X, \quad f \mapsto f(\infty)
$$


contains $U$, and is thus dominant. By composing with an appropriate automorphism of $\mathbb{P}_{k}^{1}$, it follows that, for every $c \neq 0$ in $\mathbb{P}^{1}(k)$, the evaluation morphism

$$
\mathrm{ev}_{c}: \underline{\operatorname{Hom}}\left(\left(\mathbb{P}_{k}^{1}, 0\right),(X, x)\right) \rightarrow X, \quad f \mapsto f(c)
$$

is dominant. This implies that, for every $x$ in $U(k)$, there is a covering set $f_{i}:\left(\mathbb{P}_{k}^{1}, 0\right) \rightarrow(X, x)$ for $X$, so that $X$ is geometrically-special over $k$, as required.

It is not known whether a rationally connected smooth projective variety is arithmetically-special. In fact, this is open even for Fano varieties; see [Has03] for a survey of some known results.

2.6. The case of curves. The conjectures of Campana and Lang are fully understood in the case of curves (unless one looks at orbifold curves). We state part of the optimal statement below. Note that we do not use this result, and that it is superseded by more general results on closed subvarieties of abelian varieties (see Theorem 3.5).

Theorem 2.15. Let $X$ be a smooth projective curve over $k$. Then the following statements are equivalent.

(1) The curve $X$ is of general type over $k$ (i.e., genus $(X)>1$ ).

(2) The curve $X$ is not special over $k$.

(3) The curve $X$ is geometrically hyperbolic over $k$.

(4) The curve $X$ is not geometrically special over $k$.

Proof. The equivalence of (1) and (2) for curves is well-known. The other implications follow from the definitions and the finiteness theorem of De Franchis (which says that, given smooth projective curves $C$ and $D$ with $D$ of general type, the set of non-constant morphism $C \rightarrow D$ is finite.)

2.7. Reducing to the field of complex numbers. Let $L / k$ be an extension of algebraically closed fields of characteristic zero. If $X$ is geometrically-special over $k$, then $X_{L}$ is obviously geometrically-special over $L$. The converse is probably true, but not clear to us.

Related to this is the following simple lemma which allows us to reduce certain proofs to varieties over $\mathbb{C}$ (see, for example, our proof of Theorem 1.10).

Lemma 2.16. Let $X$ be a geometrically-special variety over $k$. Then, there is a countable algebraically closed subfield $k_{0} \subset k$, and a variety $X_{0}$ over $k_{0}$ with an isomorphism $X_{0, k} \cong X$ such that $X_{0}$ is geometrically-special over $k_{0}$.

Proof. By definition, there is a dense subset $S^{\prime} \subset X(k)$ such that, for every $x$ in $S^{\prime}$, there is a pointed curve $\left(C_{x}, c_{x}\right)$ (which depends on $x$ ) and a covering set $\left(f_{x, i}:\left(C_{x}, c_{x}\right) \rightarrow(X, x)\right)_{i}$. Let $S \subset S^{\prime}$ be a countable dense subset (see Lemma 2.17 below).

For each $x \in S$, we choose a countable algebraically closed subfield $k_{x} \subset k$ such that $\left(C_{x}, c_{x}\right)$ can be defined over $k_{x}$, the variety $X$ can be defined over $k_{x}$, the point $x$ can be defined over $k_{x}$, and the covering set $\left(f_{i, x}:\left(C_{x}, c_{x}\right) \rightarrow(X, x)\right)_{i}$ can be defined over $k_{x}$. (Note that $k_{x}$ might not have finite transcendence degree, because we are descending a countably infinite amount of data.) Let $k_{0}$ be the algebraic closure (in $k$ ) of the union $\cup_{x} k_{x}$ of the countably many countable subfields $k_{x}$ of $k$. Note that $k_{0}$ is countable of characteristic zero and that $X$ has a model, say $X_{0}$, over $k_{0}$ which by construction is geometrically-special over $k_{0}$.

Lemma 2.17. Let $X$ be a variety over $k$, and let $S \subset X(k)$ be a dense subset. Then $S$ contains a countable dense subset.

Proof. Let $d:=\operatorname{dim}(X)$. For any closed subset $Z \subset X$, let the $i$-th entry of $d(Z) \in \mathbb{N}_{0}^{d+1}$ denote the number of irreducible components of $Z$ of dimension $i$. If $Z^{\prime}$ is closed in $X$ and $Z \subsetneq Z^{\prime}$, then $d(Z) \prec d\left(Z^{\prime}\right)$ with $\prec$ the lexicographic order on $\mathbb{N}_{0}^{d+1}$.

Let $\mathcal{N}:=\{d(\bar{\Sigma}) \mid \Sigma \subset S$ countable $\}$. For each $n \in \mathcal{N}$, choose a countable subset $\Sigma_{n} \subset S$ with $d\left(\overline{\Sigma_{n}}\right)=n$. Let $A:=\overline{\cup_{n} \Sigma_{n}}$. Then, as a countable union of countable sets is countable, we have that $d(A) \in \mathcal{N}$. Also, for every $n$ in $\mathcal{N}$, we have

$$
n=d\left(\overline{\Sigma_{n}}\right) \prec d(A) .
$$

If $A \neq X$, then there is an $x \in(X \backslash A)(k)$ which also lies in $S$, so that $d(A) \prec d(A \cup\{x\}) \in \mathcal{N}$. This is a contradiction. Therefore, we conclude that $A=X$, so that $\cup_{n} \Sigma_{n}$ is dense in $X$, as required. 


\section{Albanese maps}

In this section we prove that the Albanese map of a geometrically-special normal projective variety is surjective, has connected fibers, and has no multiple fibers in codimension one; see Theorem 1.10 for a precise statement.

3.1. Abelian varieties are special. We start by showing that abelian varieties are special in any sense of the word "special". We give a bit of an "overkill" proof of the fact that abelian varieties are geometricallyspecial (see Remark 3.2) for a more elementary proof.

Proposition 3.1. If $A$ is an abelian variety over $k$, then $A$ is special over $k$, arithmetically-special over $k$, and geometrically-special over $k$.

Proof. The fact that $A$ is special over $k$ was shown by Campana Cam04. By a theorem of Frey-Jarden FJ74 (see [Jav18, §3.1]), there is a subfield $K \subset k$ which is a finitely generated field extension of $\mathbb{Q}$ and a model $\mathcal{A}$ for $A$ over $K$ such that $\mathcal{A}(K)$ is dense in $A$. This shows that $A$ is arithmetically-special over $k$. Finally, to conclude the proof, let us show that $A$ is geometrically-special over $k$.

First, by Poincaré's irreducibility theorem, the abelian variety $A$ is isogenous to a finite product of simple abelian varieties. Therefore, by Lemma 2.11 and Lemma 2.13 we may and do assume that $A$ is simple. To prove the proposition, let 0 denote the origin of $A$. Since any point of $A(k)$ can be translated to 0 , it suffices to show that there is a smooth connected pointed curve $(C, c)$ and a covering set $f_{i}:(C, c) \rightarrow(A, 0)$.

If $\operatorname{dim} A=1$ (so that $A$ is an elliptic curve), define $C:=A, c=0$, and note that the graphs $\Gamma_{n}$ of the morphism (multiplication by $n$ ) $[n]: C \rightarrow A$ form a covering set which send 0 to 0 .

If $\operatorname{dim} A \geq 2$, let $C \subset A$ be a smooth irreducible curve containing $c:=0$. Since $A$ is simple, we have that $C$ is of genus at least two. In particular, by Raynaud's theorem (formerly the Manin-Mumford conjecture) Oes85, Ray83, almost all points of $C(k)$ are non-torsion. As $A$ is simple, any non-torsion point of $A$ is non-degenerate (i.e., the subgroup generated by such a point is dense in $A$ ). Now, define $f_{n}: C \rightarrow A$ by $f_{n}(c)=n \cdot c$. Then $f_{n}(0)=0$ and, as $C$ contains a dense set of non-degenerate points, the union of the graphs $\Gamma_{f_{n}}$ is dense in $C \times A$. This shows that $A$ is geometrically-special over $k$, as required.

Remark 3.2 (Referee). A referee has pointed out to us that one does not need Manin-Mumford to prove that an abelian variety $A$ is geometrically-special. Indeed, in the proof above, we only use that there exists a curve $C \subset A$ which contains infinitely many non-torsion points of $A$. To construct such a curve, one can use that the height of the set of torsion points on $A$ is bounded (with respect to any fixed embedding of $A$ into a projective space).

Proposition 3.3. If $A$ is an abelian variety over $\mathbb{C}$, then $A$ is Brody-special.

Proof. Let $\mathbb{C} \rightarrow \mathbb{C}^{\operatorname{dim} A}$ be a metrically-dense holomorphic map. If $\mathbb{C}^{\operatorname{dim} A} \rightarrow A^{\text {an }}$ is the exponential, then the image of the composed map $\mathbb{C} \rightarrow \mathbb{C}^{\operatorname{dim} A} \rightarrow A^{\text {an }}$ is Zariski-dense.

3.2. Surjectivity of the Albanese map. For a closed subvariety $V$ of an abelian variety $A$, we let $\operatorname{Sp}(V)$ be the union of translates of positive-dimensional abelian subvarieties of $A$ contained in $V$. Recall Kawamata-Ueno's theorems that $\operatorname{Sp}(V)$ is a closed subscheme of $V$, and that $\operatorname{Sp}(V) \neq V$ if and only if $V$ is of general type. We now deduce from Yamanoi's work that $V$ is geometrically hyperbolic modulo $\operatorname{Sp}(V)$.

Theorem 3.4 (Yamanoi). Let $A$ be an abelian variety over $k$. Let $X \subset A$ be a closed subvariety of $A$. Then $X$ is geometrically hyperbolic modulo $\operatorname{Sp}(X)$.

Proof. Let $\Delta_{X}^{\text {geom-hyp }}$ be the intersection over all closed subsets $\Delta \subset X$ such that $X$ is geometrically hyperbolic modulo $\Delta$ (as defined in [Jav, Section 11]). Then, by Yamanoi's theorem [Jav, Theorem 13.1] (which is a consequence of the original [Yam15, Corollary 1.1 and Corollary 1.3]), we have that $\operatorname{Sp}(X)=$ $\Delta_{X}^{g e o m-h y p}$.

Theorem 3.5 (Bloch-Ochiai-Kawamata, Faltings, Ueno, Yamanoi). Let $A$ be an abelian variety over $k$. Let $X \subset A$ be a closed integral subvariety. Then the following are equivalent.

(1) The variety $X$ is the translate of an abelian subvariety of $A$.

(2) The variety $X$ is special.

(3) If $k=\mathbb{C}$, the variety $X$ is Brody-special. 
(4) The projective variety $X$ is arithmetically-special over $k$.

(5) The variety $X$ is geometrically-special over $k$.

Proof. The implications (1) $\Longrightarrow(2),(1) \Longrightarrow(3),(1) \Longrightarrow(4)$, and (1) $\Longrightarrow$ (5) follow from Proposition 3.1 and Proposition 3.3

We show that $(5) \Longrightarrow(1)$. Assume (5) holds, and consider the Ueno fibration $f: X \rightarrow Y$ (see Uen75, Chapter IV, Theorem 10.9]); recall that this is a surjective morphism whose fibres are abelian varieties and that $Y$ is a variety of general type which can be embedded into an abelian variety. Since $Y$ is of general type and can be embedded into an abelian variety, it follows from Kawamata-Ueno's theorem that $\operatorname{Sp}(Y) \neq Y$, so that Yamanoi's theorem (Theorem 3.4) implies that $Y$ is pseudo-geometrically hyperbolic. However, since $X$ is geometrically-special and dominates the pseudo-geometrically hyperbolic variety $Y$, we have that $Y$ is zero-dimensional (Corollary 2.9). Therefore, $X$ must be the translate of an abelian subvariety of $A$.

The fact that $(2) \Longrightarrow(1)$ follows directly from Ueno's fibration theorem. One can show (3) $\Longrightarrow$ (1) and $(4) \Longrightarrow(1)$ by replacing Yamanoi's theorem above with Bloch-Ochiai-Kawamata Kaw80 and Faltings Fal94, respectively.

Corollary 3.6. If $X$ is a geometrically-special normal proper variety over $k$, then the Albanese map $X \rightarrow \operatorname{Alb}(X)$ is surjective.

Proof. Note that the image of $X$ in $\operatorname{Alb}(X)$ is geometrically-special (Lemma 2.8). Moreover, by Theorem 3.5. any geometrically-special closed (integral) subvariety of $\operatorname{Alb}(X)$ is the translate of an abelian subvariety. This implies that the image of $X \rightarrow \operatorname{Alb}(X)$ is the translate of an abelian subvariety which, by the universal property of Albanese maps, must equal $\operatorname{Alb}(X)$.

An important structure result for the Albanese map of a geometrically-special variety in the study of its fundamental group is the following corollary.

Corollary 3.7. If $X$ is a geometrically-special normal projective variety over $k$ and $Y \rightarrow X$ is a finite étale morphism, then the Albanese map of $Y$ is surjective.

Proof. By Lemma 2.11 we have that $Y$ is geometrically-special. Since $Y$ is a geometrically-special normal projective variety, its Albanese map is surjective (Corollary 3.6).

Note that Corollary 3.7 implies that the augmented irregularity of a geometrically-special normal projective variety over $k$ is bounded from above by $\operatorname{dim}(X)$.

3.3. Connectedness of the fibres. By using Kawamata's extension of the Ueno fibration theorem for closed subvarieties of abelian varieties to finite covers of abelian varieties [Kaw81, Theorem 23] and Yamanoi's recent work on the hyperbolicity of covers of abelian varieties, we can show that the Albanese map of a geometrically-special variety has connected fibres. We start with stating Kawamata's result.

Theorem 3.8 (Kawamata). Let $X$ be a normal projective variety over $k$ and let $X \rightarrow A$ be a finite morphism. Then the following data exist.

(1) An abelian subvariety $B$ of $A$;

(2) finite étale Galois covers $X^{\prime} \rightarrow X$ and $B^{\prime} \rightarrow B$;

(3) a normal projective variety $Y$ over $k$ of general type;

(4) a finite morphism $Y \rightarrow A / B$ with $A / B$ the quotient of $A$ by $B$ such that $X^{\prime}$ is a fiber bundle over $Y$ with fibers $B^{\prime}$ and with translations by $B^{\prime}$ as structure group.

Proof. See [Kaw81, Theorem 23].

We follow Yamanoi and say that a normal projective variety over $k$ is of maximal Albanese dimension if the Albanese morphism $Y \rightarrow \operatorname{Alb}(Y)$ is generically finite onto its image. The additional ingredient we need for proving the connectedness of the fibres of the Albanese map is Yamanoi's work on the hyperbolicity of varieties with maximal Albanese dimension (which builds on Theorem 3.4) .

Theorem 3.9. Let $Y$ be a normal projective variety of general type over $k$. If $Y$ is of maximal Albanese dimension, then $Y$ is pseudo-algebraically hyperbolic and pseudo-geometrically hyperbolic over $k$. 
Proof. As a pseudo-algebraically hyperbolic projective variety is pseudo-geometrically hyperbolic (Proposition 2.5), it suffices to show that $Y$ is pseudo-algebraically hyperbolic over $k$. To do so, by a standard specialization argument (see for instance [BJK] Lemma 9.2]), we may and do assume that $k=\mathbb{C}$. Then, the statement follows from Yam15, Corollary 1.(1) and Corollary 1.(3)].

Corollary 3.10. Let $X$ be a normal proper variety over $k$ of maximal Albanese dimension. Then the following statements are equivalent.

(1) The Albanese map a $: X \rightarrow \operatorname{Alb}(X)$ is birational.

(2) The variety $X$ is special.

(3) The variety $X$ is geometrically-special over $k$.

Proof. By Proposition 3.1 and the birational invariance of geometric-specialness (resp. specialness) (see Lemma 2.8), we have that $(1) \Longrightarrow(2)$ and $(1) \Longrightarrow(3)$.

Now, assume that $X$ is geometrically-special (resp. special). Then, by Corollary 3.6 (resp. Theorem 1.11), the Albanese map $a: X \rightarrow \operatorname{Alb}(X)$ is surjective. Let $X \rightarrow Z \rightarrow \operatorname{Alb}(X)$ be its Stein factorization; note that $Z$ is a normal variety over $k$ and that $Z \rightarrow \operatorname{Alb}(X)$ is a finite surjective morphism. Since $X$ is geometrically-special (resp. special) and $X \rightarrow Z$ is surjective, it follows from Lemma 2.8 that $Z$ is geometrically-special (resp. special) . We now apply Kawamata's fibration theorem (Theorem 3.8) and see that the following data exists.

(1) An abelian subvariety $B$ of $A$;

(2) finite étale Galois covers $Z^{\prime} \rightarrow Z$ and $B^{\prime} \rightarrow B$;

(3) a normal projective variety $Y$ of general type over $k$;

(4) a finite morphism $Y \rightarrow A / B$ with $A / B$ the quotient of $A$ by $B$ such that $Z^{\prime}$ is a fiber bundle over $Y$ with fibers $B^{\prime}$ and with translations by $B^{\prime}$ as structure group.

Since $Y$ is of general type, it follows from Yamanoi's theorem (Theorem 3.9) that $Y$ is pseudo-geometrically hyperbolic over $k$.

Now, since $Z$ is geometrically-special (resp. special) and $Z^{\prime} \rightarrow Z$ is finite étale, it follows from Proposition 2.12 that $Z^{\prime}$ is geometrically-special (resp. special). Since $Z^{\prime}$ is geometrically-special (resp. special) and surjects onto the variety $Y$, it follows from Lemma 2.8 that $Y$ is also geometrically-special (resp. special). Thus, since $Y$ is a geometrically-special pseudo-geometrically hyperbolic projective variety (resp. special variety of general type), we conclude that $Y$ is zero-dimensional. This implies that $Z^{\prime}=B^{\prime}$ is an abelian variety, so that the composed (finite surjective) morphism $Z^{\prime} \rightarrow Z \rightarrow \operatorname{Alb}(X)$ is finite étale. We conclude that $Z \rightarrow \operatorname{Alb}(X)$ is finite étale, so that $Z$ is an abelian variety Mum08, Section IV.18]. It now follows from the universal property of the Albanese map that $Z=\operatorname{Alb}(X)$, so that the morphism $X \rightarrow \operatorname{Alb}(X)$ has connected fibres. Finally, since $X$ has maximal Albanese dimension and the morphism $X \rightarrow \operatorname{Alb}(X)$ is surjective with connected fibres, we conclude that it is birational. This proves that $(3) \Longrightarrow(1)$ (resp. $(2) \Longrightarrow(1))$.

Corollary 3.11. If $X$ is a geometrically-special normal projective variety over $k$, then the Albanese map $X \rightarrow \operatorname{Alb}(X)$ is surjective and has connected fibres.

Proof. Let $a: X \rightarrow \operatorname{Alb}(X)$ be the Albanese map. Since $X$ is geometrically-special, the (proper) morphism $a$ is surjective (Corollary 3.6). Let $X \rightarrow Z \rightarrow \operatorname{Alb}(X)$ be its Stein factorization; note that $Z$ is a normal geometrically-special projective variety over $k$ and that $Z \rightarrow \operatorname{Alb}(X)$ is a finite surjective morphism. Clearly, $Z$ has maximal Albanese dimension, so that $Z \rightarrow \operatorname{Alb}(X)$ is birational by Corollary 3.10, and thus an isomorphism by Zariski's Main Theorem, as required.

To complete the proof of Theorem 1.10, it remains to show that the Albanese map $X \rightarrow \operatorname{Alb}(X)$ of a geometrically-special variety $X$ has no multiple fibers in codimension one. To prove this fact, we introduce geometrically-special orbifold pairs and the orbifold extension of Demailly's notion of algebraic hyperbolicity.

3.4. Orbifold pairs. A $\mathbb{Q}$-divisor $\Delta$ on a smooth projective variety $X$ over $k$ is an orbifold divisor (on $X$ ) if $\Delta=\sum_{i=1}^{r}\left(1-\frac{1}{m_{i}}\right) \Delta_{i}$, where $m_{1}, \ldots, m_{r} \geq 1$ are rational numbers and $\Delta_{1}, \ldots, \Delta_{r}$ are prime divisors; the rational number $m_{i}$ is referred to as the multiplicity of $\Delta_{i}$ in $\Delta$. A pair $(X, \Delta)$ with $X$ a smooth 
projective variety over $k$ and $\Delta=\sum_{i=1}^{r} a_{i} \Delta_{i}$ a $\mathbb{Q}$-divisor with $0 \leq a_{i} \leq 1$ is an orbifold (or orbifold-pair). Note that $\Delta$ is an orbifold divisor on $X$ in this case.

Let $\left(X, \Delta_{X}\right)$ and $\left(Y, \Delta_{Y}\right)$ be orbifold pairs. We let $m_{X}$ and $m_{Y}$ be the multiplicities of $\Delta_{X}$ and $\Delta_{Y}$, respectively. (In particular, for a prime divisor $\Delta_{i} \subset X$ contained in the support of $\Delta_{X}$, the rational number $m_{X}\left(\Delta_{i}\right)$ is the multiplicity of $\Delta_{i}$ in $\Delta_{X}$.) We define an orbifold morphism $\left(Y, \Delta_{Y}\right) \rightarrow\left(X, \Delta_{X}\right)$ to be a morphism $f: Y \rightarrow X$ of varieties over $k$ such that $f(Y) \not \subset\left\lceil\Delta_{X}\right\rceil$ and, for every prime divisor $D$ on $X$ and prime divisor $E$ on $Y$, the inequality $t \cdot m_{Y}(E) \geq m_{X}(D)$ holds, where $t$ is defined by the relation $f^{*}(D)=t \cdot E+R$ with $R$ an effective divisor not containing $E$.

Following standard conventions, we will identify a smooth projective variety $X$ with the orbifold $(X, 0)$, where 0 is the empty divisor.

In Cam11, Campana extended the notions of special variety (resp. Brody-special variety) to the setting of orbifold pairs, and then conjectured that an orbifold pair $(X, \Delta)$ over $\mathbb{C}$ is Brody-special if and only if it is special. He also extended the notion of arithmetically-special variety to the orbifold setting, and formulated the orbifold analogue of his arithmetic conjecture (Conjecture 1.6). In this section we extend the notions of geometrically-special and pseudo-geometric hyperbolicity to the setting of orbifold pairs.

Definition 3.12. An orbifold pair $(X, \Delta)$ is geometrically-special over $k$ if, for every dense open subset $U \subset X$, there exists a smooth projective connected curve $C$ over $k$, a point $c$ in $C(k)$, a point $u$ in $U(k) \backslash \Delta$, and a sequence of pairwise distinct orbifold morphisms $f_{i}: C \rightarrow(X, \Delta)$ with $f_{i}(c)=u$ for $i=1,2, \ldots$ such that $C \times X$ is covered by the graphs $\Gamma_{f_{i}}$ of these maps, i.e., the closure of $\cup_{i=1}^{\infty} \Gamma_{f_{i}}$ in $C \times X$ equals $C \times X$. (As mentioned above, we identify $C$ with the orbifold $(C, 0)$.)

Remark 3.13. Let $X$ be a smooth projective connected variety over $k$ and let $D$ be an integral divisor. For $m \in \mathbb{Z}_{\geq 1}$, define $\Delta_{m}:=\left(1-\frac{1}{m}\right) D$ and $X_{m}:=\left(X, \Delta_{m}\right)$. Then the orbifold pair $X_{1}$ is geometrically-special over $k$ if and only if $X$ is geometrically-special. Moreover, for all $m \geq 1$, we have that

$$
X_{m} \text { is geometrically-special } \Longrightarrow X_{m-1} \text { is geometrically-special }
$$

By adapting the proof of Lemma 2.7 to the orbifold setting, one obtains the following stronger statement.

Lemma 3.14. Let $(X, \Delta) \rightarrow\left(X^{\prime}, \Delta^{\prime}\right)$ be a surjective morphism of orbifold pairs. If $(X, \Delta)$ is geometricallyspecial over $k$, then $\left(X^{\prime}, \Delta^{\prime}\right)$ is geometrically-special over $k$.

A geometrically-special orbifold pair is as far away as possible from being "pseudo-geometrically hyperbolic" in the following sense (generalizing Definition 2.2).

Definition 3.15. If $(X, \Delta)$ is an orbifold pair over $k$ and $Z \subset X$ is a closed subset containing $\Delta$, then $(X, \Delta)$ is geometrically hyperbolic modulo $Z$ if, for every $x$ in $X(k) \backslash Z$, every smooth projective connected curve $C$ over $k$, and every $c$ in $C(k)$, the set of orbifold morphisms $f: C \rightarrow(X, \Delta)$ with $f(c)=x$ is finite. We say that an orbifold pair $(X, \Delta)$ over $k$ is pseudo-geometrically hyperbolic over $k$ if there is a proper closed subset $Z \subsetneq X$ containing $\Delta$ such that $(X, \Delta)$ is geometrically hyperbolic modulo $Z$.

3.5. Pseudo-algebraic hyperbolicity. We have already seen the relation between pseudo-geometric hyperbolicity and pseudo-algebraic hyperbolicity (see Proposition 2.5). In this section we extend this relation to the orbifold setting following [Rou10, Rou12.

Definition 3.16. Let $(X, \Delta)$ be an orbifold pair, and let $E \subset X$ be a closed subset. Then $(X, \Delta)$ is algebraically hyperbolic modulo $E$ over $k$ if, for every ample line bundle $L$ on $X$, there is a constant $\alpha_{X, \Delta, L, E}$ such that, for every smooth projective connected curve $C$ over $k$, and every orbifold morphism $f: C \rightarrow(X, \Delta)$ with $f(C) \not \subset \Delta \cup E$, the inequality

$$
\operatorname{deg}_{C} f^{*} L \leq \alpha_{X, \Delta, L, E} \cdot \operatorname{genus}(C)
$$

holds.

Remark 3.17 (Independence of choice of ample bundle). Let $(X, \Delta)$ be an orbifold pair over $k$, and let $E \subset X$ be a closed subset. Then $(X, \Delta)$ is algebraically hyperbolic modulo $E$ over $k$ if and only if there exists an ample line bundle $L$ on $X$ and a constant $\alpha_{X, \Delta, L, E}$ such that, for every smooth projective connected curve $C$ over $k$, and every orbifold morphism $f: C \rightarrow(X, \Delta)$ with $f(C) \not \subset E$, the inequality $\operatorname{deg}_{C} f^{*} L \leq \alpha_{X, \Delta, L, E} \cdot \operatorname{genus}(C)$. holds. 
Remark 3.18 (A big line bundle suffices). An orbifold pair $(X, \Delta)$ over $k$ is pseudo-algebraically hyperbolic over $k$ if and only if there exist a big line bundle $L$ on $X$, a proper closed subset $E \subset X$, and a constant $\alpha_{X, \Delta, L, E}$ such that, for every smooth projective connected curve $C$ over $k$, and every orbifold morphism $f: C \rightarrow(X, \Delta)$ with $f(C) \not \subset E$, the inequality $\operatorname{deg}_{C} f^{*} L \leq \alpha_{X, \Delta, L, E} \cdot \operatorname{genus}(C)$ holds. To prove this, write $L=A+F$ with $A$ ample and $F$ effective. Note that $F$ contains the augmented base locus of $L$. Then, by Remark 3.17, we have that $(X, \Delta)$ is algebraically hyperbolic modulo the proper closed subset $E \cup F$.

The following proposition generalizes Proposition 2.5 to the setting of orbifold pairs under the additional assumption that the rational curves on the space $X$ are contained in a proper closed subset.

Proposition 3.19. Let $(X, \Delta)$ be an orbifold pair and let $E \subset X$ be a closed subset such that every nonconstant morphism $\mathbb{P}_{k}^{1} \rightarrow X$ factors over $E$. If $(X, \Delta)$ is algebraically hyperbolic modulo $E$, then $(X, \Delta)$ is geometrically-hyperbolic modulo E.

Proof. We may and do assume that $E \neq X$. Suppose that $(X, \Delta)$ is not geometrically-hyperbolic modulo $E$. Then, we can choose a smooth proper connected curve $C$ over $k$, a point $c \in C(k)$, a point $x \in X(k) \backslash E \cup \Delta$, and an infinite sequence of orbifold morphisms $f_{i}: C \rightarrow(X, \Delta)$ with $f_{i}(c)=x$. Note that, as $x \in X(k) \backslash E$, we have that $f_{i}(C) \not \subset E$. Therefore, if $L$ is an ample line bundle, since $X$ is algebraically-hyperbolic modulo $E$, there is a real number $\alpha$ depending only on $X, \Delta, E, L$ such that, for every $i=1,2, \ldots$, the inequality

$$
\operatorname{deg}_{C} f_{i}^{*} L \leq \alpha \cdot \operatorname{genus}(C)
$$

holds. Thus, replacing $\left(f_{i}\right)_{i=1}^{\infty}$ by a suitable subsequence if necessary, we may and do assume that $d:=$ $\operatorname{deg}_{C} f_{i}^{*} L$ is independent of $i$. Then, the component $\underline{\operatorname{Hom}}_{k}^{d}((C, c),(X, x))$ parametrizing pointed morphisms $(C, c) \rightarrow(X, x)$ over $k$ of degree $d$ is a positive-dimensional variety over $k$ (as it is quasi-projective and has infinitely many elements). This implies by Mori's bend-and-break that $X$ has a rational curve passing through $x$ (see Deb01, Proposition 3.1]), contradicting the fact that every rational curve in $X$ is contained in $E$.

Definition 3.20. An orbifold pair $(X, \Delta)$ is pseudo-algebraically hyperbolic over $k$ if there is a proper closed subset $E \subsetneq X$ containing $\Delta$ such that $(X, \Delta)$ is algebraically hyperbolic modulo $E$.

3.6. Lang's conjecture for orbifold divisors on abelian varieties. The following result of Yamanoi confirms the expectation that abelian varieties endowed with a big orbifold divisor are pseudo-algebraically hyperbolic in the orbifold sense.

Theorem 3.21 (Yamanoi). Let $\Delta$ be a big orbifold divisor on an abelian variety $A$ over $k$. Then, the orbifold pair $(A, \Delta)$ is pseudo-algebraically hyperbolic over $k$.

Proof. We may and do assume that $k=\mathbb{C}$. Let us show how the result follows from Yamanoi's theorem Yam15, Corollary 2].

Write $\Delta=\sum_{i=1}^{r}\left(1-\frac{1}{m_{i}}\right) D_{i}$, where $m_{1}, \ldots, m_{r}>1$ are rational numbers and $D_{1}, \ldots, D_{r}$ are integral divisors, respectively. Let $L$ be an ample line bundle on $A$. Now, since $\Delta$ is big, we may choose a rational number $\epsilon>0$ and an effective divisor $F$ such that $\Delta-F-\epsilon L$ is ample, so that $\Delta-\epsilon L$ is big.

With these choices made, we now apply Yamanoi's theorem Yam15, Corollary 2] to the integral divisors $D_{i}$ and see that, for every $i=1, \ldots, r$, there is a proper closed subset $E_{i}:=\Sigma\left(A, D_{i}, L\right)$ and a positive real number $a_{i}=a_{i}\left(A, D_{i}, L\right)$ such that, for every smooth projective connected curve $C$ over $k$ and every morphism of orbifold pairs $f: C \rightarrow(A, \Delta)$ with $f(C) \not \subset E_{i} \cup \Delta$, the inequality

$$
\operatorname{deg} f^{*} D_{i} \leq \# f^{-1}\left(D_{i}\right)+a_{i} \cdot \operatorname{genus}(C)+\frac{\epsilon}{r} \operatorname{deg} f^{*} L
$$

holds. Since $f: C \rightarrow(A, \Delta)$ is a morphism of orbifold pairs, we have that

$$
\# f^{-1}\left(D_{i}\right) \leq \frac{1}{m_{i}} \operatorname{deg} f^{*} D_{i} .
$$

This implies, for every $i=1, \ldots, r$, that

$$
\operatorname{deg} f^{*} D_{i} \leq \frac{1}{m_{i}} \operatorname{deg} f^{*} D_{i}+a_{i} \cdot \operatorname{genus}(C)+\frac{\epsilon}{r} \operatorname{deg} f^{*} L
$$


Thus, for every morphism of orbifold pairs $f: C \rightarrow(A, \Delta)$ with $f(C) \not \subset \cup_{i=1}^{r} E_{i} \cup \Delta$,

$$
\sum_{i=1}^{r}\left(\operatorname{deg} f^{*} D_{i}-\frac{1}{m_{i}} \operatorname{deg} f^{*} D_{i}\right) \leq \sum_{i=1}^{r}\left(a_{i} \cdot \operatorname{genus}(C)+\frac{\epsilon}{r} \operatorname{deg} f^{*} L\right) .
$$

Defining $a:=\sum_{i=1}^{r} a_{i}$, we obtain that

$$
\operatorname{deg} f^{*}(\Delta-\epsilon L) \leq a \cdot \operatorname{genus}(C) .
$$

Since $\Delta-\epsilon L$ is big, we conclude that $(A, \Delta)$ is algebraically hyperbolic modulo $E$ (Remark 3.18), as required.

Theorem 3.22 (Yamanoi $+\epsilon$ ). Let $\Delta$ be a big orbifold divisor on an abelian variety $A$ over $k$. Then, the orbifold pair $(A, \Delta)$ is pseudo-geometrically-hyperbolic over $k$.

Proof. By Yamanoi's theorem (Theorem 3.21), we have that $(A, \Delta)$ is pseudo-algebraically hyperbolic over $k$. Therefore, as $A$ has no rational curves, the result follows from Proposition 3.19.

Theorem 3.24 below provides a converse to Theorem 3.22 and may be considered as a version of Lang's conjecture (Conjecture 1.2) for orbifold pairs on abelian varieties. We include it for the sake of future reference.

Lemma 3.23. Let $(X, \Delta)$ be an orbifold pair over $k$, and let $E \subset X$ be a proper closed subset. If $(X, \Delta)$ is geometrically hyperbolic modulo $E$ over $k$ and $A$ is an abelian variety over $k$, then every non-constant orbifold morphism $A \rightarrow(X, \Delta)$ factors over $E$.

Proof. Let $\varphi: A \rightarrow(X, \Delta)$ be a non-constant orbifold morphism. Let $0 \in A$ be the origin and assume $\varphi(0) \notin E$. Let $X^{\prime}=\varphi(A)$, and note that $X^{\prime}$ is geometrically hyperbolic modulo the proper closed subset $E^{\prime}:=E \cap X^{\prime}$. Since $A$ is geometrically-special (Proposition 3.1), it follows that $X^{\prime}$ is geometrically-special (Lemma 2.7). Since $X^{\prime}$ is geometrically-special and pseudo-geometrically hyperbolic, we conclude that $X^{\prime}$ is zero-dimensional (Corollary 2.9), so that $\varphi$ is constant, as required.

Theorem 3.24. Let $\Delta$ be an orbifold divisor on an abelian variety $A$ over $k$. Then, the orbifold pair $(A, \Delta)$ is of general type if and only if $(A, \Delta)$ is pseudo-geometrically hyperbolic over $k$.

Proof. Since $\omega_{A}=\mathcal{O}_{A}$, the orbifold pair $(A, \Delta)$ is of general type if and only if $\Delta$ is big. Thus, if $(A, \Delta)$ is of general type, then $(A, \Delta)$ is pseudo-geometrically hyperbolic over $k$ by Theorem 3.22

Conversely, assume that $(A, \Delta)$ is not of general type. Let $E \subset A$ be a closed subset such that $(A, \Delta)$ is geometrically hyperbolic modulo $E$. To conclude the proof, it suffices to show that $E=A$.

Note that $\Delta$ is not big. Let $\Delta^{\prime}$ be a non-big component of $\Delta$. (As the sum of big divisors is big, such a component exists.) Then, we have a non-zero abelian variety $B$, a homomorphism $f: A \rightarrow B$, and a big orbifold divisor $D$ on $B$ such that $\Delta^{\prime} \subset f^{-1}(\operatorname{Supp}(D))$. Thus, for every $t$ in $B \backslash D$, the fibre $A_{t}$ of $f: A \rightarrow B$ is a positive-dimensional abelian subvariety of $A$. Note that, as $A_{t}$ and $\Delta^{\prime}$ are disjoint, we have that the natural inclusion $A_{t} \rightarrow A \backslash \Delta^{\prime}$ induces an orbifold inclusion $A_{t} \subset\left(A, \Delta^{\prime}\right)$. Since $\left(A, \Delta^{\prime}\right)$ is geometrically hyperbolic modulo $E$, by Lemma 3.23 the inclusion $A_{t} \subset A$ factors over $E$. This implies that the induced morphism $E \rightarrow B$ is dominant with general fibres of dimension $\operatorname{dim} A_{t}$, so that $\operatorname{dim} E=\operatorname{dim} A_{t}+\operatorname{dim} B=\operatorname{dim} A$. This implies that $E=A$, as required.

3.7. The Albanese map has no multiple fibres. In this section we complete the proof of our main result on the Albanese map of a geometrically-special normal projective variety (Theorem 1.10). To prove Theorem 1.10, we will make use of the "orbifold base" (as defined below) of the Albanese fibration.

Definition 3.25. Let $f: X \rightarrow Y$ be a surjective morphism of normal projective varieties over $k$ with connected fibres. We define the orbifold divisor $\Delta(f)$ on $Y$ as follows. Let $D \subset Y$ be a prime divisor of $Y$. Let $F_{1}, \ldots, F_{r}$ be the irreducible divisors of $X$ which map surjectively to $D$ via $f$. Then, we may write the scheme-theoretic fibre of $f$ over $D$ as $f^{*} D=R+\sum_{k} t_{k} \cdot F_{k}$ with $R$ an $f$-exceptional divisor of $X$ with $f(R) \subsetneq D$. For each irreducible Weil divisor $D \subset Y$, we define

$$
m_{f}(D):=\inf \left\{t_{k}\right\}
$$

We define $\Delta:=\sum_{D}\left(1-\frac{1}{m_{f}(D)}\right) D$, where the sum runs over all prime divisors of $Y$. 
Proof of Theorem 1.10. We first reduce to $k=\mathbb{C}$ (so that we can appeal to Yamanoi's work Yam15). To do so, choose a countable algebraically closed subfield $k_{0} \subset k$ such that there is a normal projective variety $X_{0}$ over $k_{0}$ with $X_{0, k} \cong X$ such that $X_{0}$ is geometrically special over $k_{0}$ (Lemma 2.16). Since $k_{0}$ is countable, we may choose an embedding $k_{0} \rightarrow \mathbb{C}$. Since $X_{0}$ is geometrically-special over $k_{0}$, we have that $X_{0, \mathbb{C}}$ is geometrically-special over $\mathbb{C}$. By standard properties of the Albanese map, it suffices to prove the theorem for $X_{0, \mathbb{C}}$. Thus, to prove the theorem, we may and do assume that $k=\mathbb{C}$.

Let $X$ be a normal proper geometrically-special variety over $k$, and let alb : $X \rightarrow \operatorname{Alb}(X)$ be the Albanese map. By Corollary 3.11, the morphism alb is surjective with connected fibres. Therefore, we may consider the associated orbifold divisor $\Delta(\mathrm{alb}) \subset \operatorname{Alb}(X)$ of alb (Definition 3.25). To prove the theorem, it suffices to show that alb has no multiple fibers in codimension one (Definition [1.9).

We argue by contradiction and suppose that alb has a multiple fiber in codimension one. In particular, the orbifold divisor $\Delta(\mathrm{alb})$ is non-empty.

Let $\Delta^{\prime}$ be an irreducible component of the support of $\Delta(\mathrm{alb})$. Then, by Ueno's fibration theorem Uen75, Chapter IV, Theorem 10.9], there is a non-zero abelian variety $A$, a homomorphism $F: \operatorname{Alb}(X) \rightarrow A$, and a big divisor $D$ on $A$ such that $\Delta^{\prime}=F^{-1}(\operatorname{Supp} D)$. (Explicitly: $D$ is defined to be the general type base of the Ueno fibration for $\Delta^{\prime}$. The fact that $D$ is a big divisor follows from the fact that $D$ is of general type, so that it is in particular a divisor with finite stabilizer. Such divisors are ample [BG06, Lemma 8.5.6]).

We now consider the composed morphism $f: X \rightarrow \operatorname{Alb}(X) \rightarrow A$ and the orbifold divisor $\Delta=\left(1-\frac{1}{m}\right) D$, where $m$ is the multiplicity of $D$ in the orbifold divisor $\Delta(f)$ of $f$. If $m=1$, then the fibre over $D$ has a component of multiplicity one. In particular, if $m=1$, the fibre of alb over $\Delta^{\prime}$ also has a component of multiplicity one. (As the referee pointed out to us, this component might be alb-exceptional a priori, so that $\Delta$ (alb) might still be non-trivial; see Remark 3.28) Thus, to prove the theorem, it suffices to show that $m=1$. We argue by contradiction and assume that $m>1$.

Note that $f$ is surjective with connected fibres (and that $X \rightarrow(A, \Delta)$ might not be orbifold). Write $f^{*} D=R+\sum_{k=1}^{r} t_{k} F_{k}$, where $F_{1}, \ldots, F_{r}$ are the irreducible divisors in $f^{-1}(D)$ mapping surjectively onto $D$ and $R$ is an $f$-exceptional divisor of $X$ with $f(R) \subsetneq D$. Let $Z$ be the image of $R$ in $A$ and note that $Z$ is of codimension at least two (and possibly empty) in $A$.

Since $X$ is geometrically-special over $k$, we may (and do) choose a covering set $g_{i}:(C, c) \rightarrow(X, x)$ with $x \notin f^{-1}(D)$ and $C$ a smooth projective curve over $k$. Let $\pi_{C}: C \rightarrow \mathbb{P}_{k}^{1}$ be a finite morphism of degree at most genus $(C)+1$ and define $C^{0}=\pi_{C}^{-1}\left(\mathbb{A}_{k}^{1}\right)$. Consider the covering set $f_{i}:(C, c) \rightarrow(A, a)$, where $f_{i}:=f \circ g_{i}$ and $a:=f(x)$. Note that $f_{i}(C) \not \subset D$ and that $f_{i}$ is "orbifold modulo $Z$ " in the sense that the desired inequality on multiplicities holds outside of $Z$. More precisely, we have

$$
\# f_{i}^{-1}(D)=\# f_{i}^{-1}(D \backslash Z)+\# f_{i}^{-1}(Z) \leq \frac{1}{m} \operatorname{deg} f_{i}^{*} D+\# f_{i}^{-1}(Z) .
$$

Let $L$ be an ample line bundle on $A$ and let $\epsilon>0$ be a rational number such that $\Delta-2 \epsilon L$ is big. Applying [Yam15, Proposition 5] (with " $S$ " in loc. cit. a point), we obtain that there is a real number $\kappa=\kappa(C, A, Z, L, \epsilon)>0$ depending only on $C, A, Z, L, \epsilon$ and a finite set $P=P(A, Z, L, \epsilon)$ of positivedimensional abelian subvarieties of $A$ depending only on $A, Z, L, \epsilon$ such that either

$$
\# f_{i}^{-1}(Z) \leq \kappa+\epsilon \cdot \operatorname{deg} f_{i}^{*} L
$$

or $f_{i}(C)$ is contained in the subset $\Phi:=\cup_{B \in P} \Phi(B, Z) \subset A$, where

$$
\Phi(B, Z):=\{x \in A \mid \operatorname{dim}((x+B) \cap Z) \geq \operatorname{dim} B-1\} .
$$

Note that $\Phi(A, Z)=\emptyset$ and that $\Phi$ is a proper closed subset of $A$.

Since $\left(f_{i}\right)_{i}$ is a covering set, replacing $\left(f_{i}\right)_{i}$ by a subsequence if necessary, we may and do assume that, for each $i$, we have that $f_{i}(C) \not \subset \Phi$. In particular, for each $i$, we have

$$
\# f_{i}^{-1}(Z) \leq \kappa+\epsilon \cdot \operatorname{deg} f_{i}^{*} L \text {. }
$$

Now, as in the proof of Theorem 3.21, it follows from [Yam15, Corollary 2] that there is a real number $\kappa_{2}$ depending only on $C, A, D, L, \epsilon$ such that

$$
\operatorname{deg} f_{i}^{*} D \leq \# f_{i}^{-1}(D)+\kappa_{2}+\epsilon \cdot \operatorname{deg} f_{i}^{*} L
$$


Substituting inequalities (3.1) and (3.4) in (3.5), we see that

$$
\begin{aligned}
\operatorname{deg} f_{i}^{*} D & \leq \# f_{i}^{-1}(D)+\kappa_{2}+\epsilon \cdot \operatorname{deg} f_{i}^{*} L \\
& \leq \frac{1}{m} \operatorname{deg} f_{i}^{*} D+\kappa+\epsilon \cdot \operatorname{deg} f_{i}^{*} L+\kappa_{2}+\epsilon \cdot \operatorname{deg} f_{i}^{*} L
\end{aligned}
$$

Since $\Delta=\left(1-\frac{1}{m}\right) D$, we may rewrite this as

$$
\operatorname{deg} f_{i}^{*}(\Delta-2 \epsilon L) \leq \kappa+\kappa_{2} .
$$

Note that, as $m>1$, the divisor $\Delta-2 \epsilon L$ is big. Therefore, as in Remark 3.18, since $\Delta-2 \epsilon L$ is big, we may write $\Delta-2 \epsilon L=M+F$, where $M$ is ample and $F$ is effective. Replacing the covering set $\left(f_{i}\right)_{i}$ by a subsequence if necessary, we may and do assume that $f_{i}(C) \not \subset F$ for every $i$. Then, for every $i$, we have that

$$
\operatorname{deg} f_{i}^{*} M \leq \operatorname{deg} f_{i}^{*} M+\operatorname{deg} f_{i}^{*} F=\operatorname{deg} f_{i}^{*}(\Delta-2 \epsilon L) \leq \kappa+\kappa_{2} .
$$

Since $\kappa+\kappa_{2}$ is a real number depending only on $C, A, D, L$, $\epsilon$, we conclude that the moduli space of pointed maps $(C, c) \rightarrow(A, a)$ is positive-dimensional (as it has a component containing an infinite subsequence of $\left.\left(f_{i}\right)_{i}\right)$. Therefore, by Mori's bend-and-break (see Deb01, Proposition 3.1]), there is a rational curve in $A$ (passing throug $a$ ), contradicting the fact that $A$ has no rational curves. This contradiction concludes the proof.

Remark 3.26. In an earlier version of our paper, we used that the map alb : $X \rightarrow(\operatorname{Alb}(X), \Delta(\operatorname{alb}))$ is orbifold in the proof of Theorem 1.10. This is however a priori false, as a referee pointed out to us. The issue comes from alb-exceptional components over $\Delta$. We deal with this "codimension two" issue by using [Yam15, Proposition 5] in our proof.

Remark 3.27 (Analytic analogue). As already mentioned in the introduction, the analytic analogue of Theorem [1.10 was known before. Indeed, as stated in [CC16. Proposition 5.1], for $X$ a Brody-special normal projective variety, the Albanese map is surjective, has connected fibres, and has no multiple fibres in codimension one; this can be deduced directly from [NWY07, Main Theorem], [LW12, Corollary 1.4], and [Kaw81, Theorem 13]. The codimension two phenomenon discussed in Remark [3.26] also occurs in the proof of the analytic analogue and is dealt with accordingly in the proof of [LW12, Theorem 4.15]. Note that in our proof of Theorem 1.10 we can not appeal to [LW12, as they only deal with holomorphic maps from $\mathbb{C}$ to $X$. This is why we resort to Yamanoi's results in the above proof.

Remark 3.28. Given a surjective morphism with connected fibres $X \rightarrow Y$, there is a subtle difference between "having no multiple fibres in codimension one" and having a trivial orbifold divisor. We stress that we do not show that the Albanese fibration $X \rightarrow \operatorname{Alb}(X)$ of a geometrically-special has trivial orbifold divisor (or is "base-special"). Indeed, our proof above only shows that the orbifold base of $f: X \rightarrow$ $\operatorname{Alb}(X) \rightarrow A$ is trivial. Since a multiplicity one component of the fibre of $f$ over a codimension one point might be alb-exceptional, one can not conclude that the Albanese fibration has trivial orbifold divisor. This subtle issue seems to have been overlooked in [Cam04, Proposition 5.3], CC16, Proposition 5.1] and LW12.

\section{Virtually abelian fundamental Groups}

In this section we show that the image of the fundamental group of a geometrically-special smooth projective variety over $\mathbb{C}$ along a linear representation is virtually abelian (Theorem 1.15). Our proof follows closely Yamanoi's strategy [Yam10], and we will indicate as carefully as possible how to adapt Yamanoi's line of reasoning to prove Theorem 1.15.

The key observation is that the formal properties of Brody-special varieties used by Yamanoi also hold for geometrically-special varieties (e.g., the Albanese map of a Brody-special proper variety is surjective, much like the Albanese map of a geometrically-special proper variety). The novel technical result is arguably Theorem 4.1 
4.1. The case of $p$-unbounded representations. It is reasonable to state that Yam10, Proposition 3.1] is one of the crucial results of Yamanoi's paper on fundamental groups of Brody-special varieties. We start with establishing the geometrically-special analogue of his result; see Proposition 4.2 for a precise statement.

Recall that, if $L$ is a field, a group scheme $G$ over $L$ is an almost simple algebraic group over $L$ if $G$ is a non-commutative smooth affine (geometrically) connected group scheme over $L$ and $G$ has no infinite proper closed normal subgroups.

If $p$ is a prime number and $G$ is an almost simple algebraic group over a finite field extension $K$ of $\mathbb{Q}_{p}$, then we say that a representation $\rho: \pi_{1}(X) \rightarrow G(K)$ is $p$-bounded if its image $\rho\left(\pi_{1}(X)\right)$ is contained in a maximal compact subgroup of $G(K)$. If $\rho$ is not $p$-bounded, then we say that $\rho$ is $p$-unbounded.

In what follows we will prove that the fundamental group of a smooth projective geometrically-special variety does not admit a Zariski-dense representation into an almost simple algebraic group. To do so, we will argue case-by-case. The crucial (arguably most technical) case is that of a big p-unbounded representation considered first. (For the reader's convenience, we recall that a representation $\rho: \pi_{1}(X) \rightarrow$ $\mathrm{GL}_{n}(\mathbb{C})$ is big if there is a countable collection of proper closed subsets $X_{i} \subsetneq X$ with $i=1, \ldots$ such that, for every positive-dimensional subvariety $Z \subset X$ containing a point of $X \backslash \cup_{i=1}^{\infty} X_{i}$, the group $\rho\left[\operatorname{Im}\left(\pi_{1}(\tilde{Z}) \rightarrow\right.\right.$ $\left.\left.\pi_{1}(X)\right)\right]$ is infinite, where $\tilde{Z} \rightarrow Z$ is a desingularization of $Z$.)

Theorem 4.1. Let $X$ be a smooth projective variety over $\mathbb{C}$, and let $K$ be a finite extension of $\mathbb{Q}_{p}$. Let $G$ be an almost simple algebraic group over $K$. Assume that there exists a big p-unbounded representation $\rho: \pi_{1}(X) \rightarrow G(K)$ whose image is Zariski-dense in $G$. Then $X$ is not geometrically-special over $\mathbb{C}$.

Proof. As in Yamanoi's proof of [Yam10, Proposition 3.1] we will use Zuo's results on spectral coverings [Zuo96, Zuo99] (see also [CCE15]). For instance, by Zuo's work (Zuo96, Theorem 1] and [Zuo96, Theorem 2]), as $\rho$ is big, the variety $X$ is "Chern-hyperbolic". In particular, there is a proper closed subset $\Delta \subsetneq X$ such that every non-constant morphism $f: \mathbb{P}_{\mathbb{C}}^{1} \rightarrow X$ factors over $\Delta$, i.e., we have $f\left(\mathbb{P}_{\mathbb{C}}^{1}\right) \subset \Delta$. Moreover, the variety $X$ is of general typ 1 .

We will make explicit use of Zuo's spectral covering $\pi: X^{s} \rightarrow X$; see Yam10, p 554] for an explanation of the notation. As is explained in loc. cit., the variety $X^{s}$ is equipped with holomorphic one-forms $\omega_{1}, \ldots, \omega_{\ell}$ that are contained in $\mathrm{H}^{0}\left(X^{s}, \pi^{*} \Omega_{X}^{1}\right)$ and such that $\pi: X^{s} \rightarrow X$ is unramified outside $\bigcup_{\omega_{i} \neq \omega_{j}}\left(\omega_{i}-\omega_{j}\right)_{0}$. Moreover, the forms $\omega_{1}, \ldots, \omega_{\ell}$ are used to construct the adapted Albanese map $\alpha: X^{s} \rightarrow A$. We note that thanks to Zuo96, the morphism $\alpha$ is generically finite onto its image, so that $X^{s}$ has maximal Albanese dimension (because the adapted Albanese map factorizes through the Albanese map). Moreover, since $X$ is of general type, it follows that $X^{s}$ is of general type. Therefore, it follows from Yamanoi's theorem (Theorem 3.9) that $X^{s}$ is pseudo-algebraically hyperbolic. In particular, replacing $\Delta \subset X$ by a suitable larger proper closed subset, we may assume that $X^{s}$ is algebraically hyperbolic modulo $\pi^{*} \Delta$. Now, we fix an ample line bundle $M$ on $X$, so that (by the algebraic hyperbolicity of $X^{s}$ modulo $\pi^{*} \Delta$ ) there is a real number $\gamma$ depending only on $X, M$, and $\pi^{*} \Delta$ such that, for every smooth projective connected curve $W$ over $\mathbb{C}$ and every morphism $g: W \rightarrow X^{s}$ with $g(W) \not \subset \pi^{*} \Delta$, the inequality

$$
\operatorname{deg} g^{*}\left(\pi^{*} M\right) \leq \gamma \cdot \operatorname{genus}(W)
$$

holds.

To prove the proposition, we argue by contradiction. Thus, assume that $X$ is geometrically-special over $\mathbb{C}$. Define $U:=X \backslash \Delta$, choose a smooth projective connected curve $C$ of genus at least two, a point $c_{0}$ in $C$, a point $x$ in $U$, and a covering set of non-constant morphisms $f_{k}:\left(C, c_{0}\right) \rightarrow(X, x)$ with $k=1, \ldots$ By the definition of a covering set, replacing the sequence of morphisms $\left(f_{k}\right)_{k=1}^{\infty}$ by a suitable subsequence if necessary, we have that, for every proper closed subset $Z \subset X$, there are only finitely many integers $k$ such that $f_{k}(C) \subset Z$.

For every $k=1, \ldots$, we consider the pull-back $V_{k} \rightarrow X^{s}$ of $f_{k}: C \rightarrow X$ along $\pi: X^{s} \rightarrow X$. Let $W_{k}$ be the normalization of $V_{k}$, and let $g_{k}: W_{k} \rightarrow X^{s}$ denote the composed morphism $W_{k} \rightarrow V_{k} \rightarrow X^{s}$. (Note that $W_{k}$ is a, possibly disconnected, smooth projective curve over $\mathbb{C}$.) This gives us, for every $k=1, \ldots$,

\footnotetext{
${ }^{1}$ At this point, it is clear that $X$ is not special. However, proving that $X$ is not geometrically-special requires many more arguments.
} 
the following commutative diagram

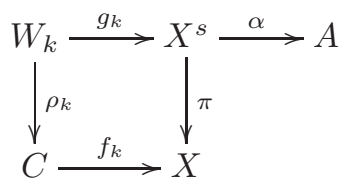

Now, since $f_{k}\left(c_{0}\right)=x \notin \Delta$, for every connected component $W_{k}^{\prime}$ of $W_{k}$, we have that $g_{k}\left(W_{k}^{\prime}\right) \not \subset \pi^{*} \Delta$. In particular, using that $X^{s}$ is algebraically hyperbolic modulo $\pi^{*} \Delta$ and letting genus $\left(W_{k}\right)$ denote $\sum \operatorname{genus}\left(W_{k}^{\prime}\right)$ where the sum runs over all connected components $W_{k}^{\prime}$ of $W_{k}$, we see that the inequality

$$
\operatorname{deg} \pi \cdot \operatorname{deg} f_{k}^{*} M=\operatorname{deg}\left(f_{k} \circ \rho_{k}\right)^{*} M=\operatorname{deg} g_{k}^{*}\left(\pi^{*} M\right) \leq \gamma \cdot \operatorname{genus}\left(W_{k}\right)
$$

holds; see (4.1) above. Note that we used that $\operatorname{deg} \rho_{k}=\operatorname{deg} \pi$.

Let $D \subset X^{s}$ be the (support of the) ramification divisor of $\pi: X^{s} \rightarrow X$. Then $D \subset \bigcup_{1 \leq i<j<\ell} \Xi_{i j}$, where $\Xi_{i j}:=\left(\omega_{i}-\omega_{j}\right)_{0}$. Let $\omega_{i j}:=\omega_{i}-\omega_{j}$. The idea is to use the forms $\omega_{i}$ to "control" the genus of $W_{k}$ in terms of the degree of $g_{k}$. (Since there is no a priori control on the branch points of $W_{k} \rightarrow C$, the genus of $W_{k}$ may tend to infinity as $k$ tends to infinity.)

To do so, by the Riemann-Hurwitz formula $K_{W_{k}}=\rho_{k}^{*}\left(K_{C}\right)+R_{k}$, it suffices to "control" the degree of the ramification divisor $R_{k}$ of $\rho_{k}$. Note that

$$
\operatorname{deg} R_{k} \leq\left(\operatorname{deg} \rho_{k}\right)\left(\# g_{k}^{-1}(D)\right) \leq\left(\operatorname{deg} \rho_{k}\right) \sum_{1 \leq i<j \leq \ell} \# g_{k}^{-1}\left(\Xi_{i j}\right) .
$$

In particular,

$$
\operatorname{genus}\left(W_{k}\right) \leq\left(\operatorname{deg} \rho_{k}\right) \cdot \operatorname{genus}(C)+\left(\operatorname{deg} \rho_{k}\right) \sum_{1 \leq i<j \leq \ell} \# g_{k}^{-1}\left(\Xi_{i j}\right) .
$$

Let $1 \leq i<j \leq \ell$ be such that $g_{k}^{*} \omega_{i j} \neq 0$. Consider $\beta$ a holomorphic section of $K_{C}$ and the meromorphic function $\eta=g_{k}^{*} \omega_{i j} / \rho_{k}^{*} \beta$. We can consider $g_{k}^{*} \omega_{i j}$ as a holomorphic section of $\rho_{k}^{*} \Omega_{C}$, so that $\operatorname{deg}(\eta=\infty)=$ $\left(\operatorname{deg} \rho_{k}\right) \operatorname{deg} K_{C}$. Therefore, it follows that

$$
\begin{aligned}
\# g_{k}^{-1}\left(\Xi_{i j}\right) & \leq \operatorname{deg}(\eta=0)=\operatorname{deg}(\eta=\infty) \\
& \leq(\operatorname{deg} \pi) \operatorname{deg} K_{C} \leq 2(\operatorname{deg} \pi) \operatorname{genus}(C) .
\end{aligned}
$$

Now, let $1 \leq i<j \leq \ell$ be such that $g_{k}^{*} \omega_{i j}=0$. Following Yamanoi (see Yam10 p.557 for details), we consider the Albanese map $b: X^{s} \rightarrow B$ with respect to $\omega_{i j}$. Also, we let $S \rightarrow b\left(X^{s}\right)$ be the normalization of $b\left(X^{s}\right)$, so that $b$ factors as

$$
X^{s} \stackrel{c}{\longrightarrow} S \stackrel{\psi}{\longrightarrow} B
$$

Then, the image $c\left(\Xi_{i j}\right)$ of $\Xi_{i j}$ is a point $P$ in $S$. Let $L$ be an ample line bundle on $S$. Then, Yamanoi constructs (see Yam10 p.558 for details), for every positive integer $n$, a positive integer $l_{n}$ and a divisor $D_{n}$ on $S$ such that the following three properties hold.

(1) The sequence of integers $\left(l_{n}\right)_{n=1}^{\infty}$ is strictly increasing and satisfies $\lim _{n \rightarrow \infty} \frac{l_{n}}{n}=0$;

(2) The divisor $D_{n}$ is contained in $\left|L^{\otimes l_{n}}\right|$;

(3) If $c \circ g_{k}(y)=P$, then $\operatorname{ord}_{y}\left(c \circ g_{k}\right)^{*} D_{n} \geq n$.

Now, since $\pi^{*} M$ is ample, we may choose an integer $N_{0}$ such that $N_{0} \pi^{*} M-c^{*} L \geq 0$. Next, we choose an integer $n$ such that $\frac{l_{n}}{n}<\frac{1}{(\ell+1)^{3}}$; such an integer exists as $l_{n}=o(n)$ (see (1) above). Now, by our choice of covering set $\left(f_{k}\right)_{k=1}^{\infty}$, there is an integer $k_{i, j}$ such that, for all $k \geq k_{i, j}$, we have that $f_{k}(C) \not \subset \pi\left(c^{-1}\left(D_{n}\right)\right)$. In particular, for all $k \geq k_{i, j}$, we have that $c \circ g_{k}\left(W_{k}\right) \not \subset D_{n}$. Therefore, for $k \geq k_{i, j}$, we have that

$$
\begin{aligned}
\# g_{k}^{-1}\left(\Xi_{i j}\right) & \leq \#\left(c \circ g_{k}\right)^{-1}(P) \leq \frac{1}{n} \operatorname{deg}\left(c \circ g_{k}\right)^{*} D_{n} \leq \frac{l_{n}}{n} \operatorname{deg}\left(c \circ g_{k}\right)^{*} L \\
& \leq \frac{1}{(\ell+1)^{3}} \operatorname{deg}\left(c \circ g_{k}\right)^{*} L \leq \frac{N_{0}}{(\ell+1)^{3}} \cdot \operatorname{deg} g_{k}^{*} \pi^{*} M
\end{aligned}
$$

Define

$$
k_{0}=\max \left(k_{i, j} \mid 1 \leq i<j \leq \ell \text { such that } g_{k}^{*} \omega_{i j} \neq 0\right\} .
$$


By combining (4.7) with (4.6) and (4.5), we obtain that, for all $k \geq k_{0}$,

$$
\begin{aligned}
\operatorname{genus}\left(W_{k}\right) & \leq\left(\operatorname{deg} \rho_{k}\right) \cdot \operatorname{genus}(C)+\left(\operatorname{deg} \rho_{k}\right) \sum_{1 \leq i<j \leq \ell} \# g_{k}^{-1}\left(\Xi_{i j}\right) \\
& \leq\left(\operatorname{deg} \rho_{k}\right) \cdot\left(\operatorname{genus}(C)+\sum_{\substack{1 \leq i<j \leq \ell \\
g_{k}^{*} \omega_{i j} \neq 0}}\left(\# g_{k}^{-1}\left(\Xi_{i j}\right)\right)+\sum_{\substack{1 \leq i<j \leq \ell \\
g_{k}^{*} \omega_{i j}=0 .}}\left(\# g_{k}^{-1}\left(\Xi_{i j}\right)\right)\right) \\
& \leq 3(\operatorname{deg} \pi)^{2} \ell^{2} \cdot \operatorname{genus}(C)+\frac{\ell^{2} N_{0}}{(\ell+1)^{3}} \operatorname{deg} g_{k}^{*} \pi^{*} M \\
& =3(\operatorname{deg} \pi)^{2} \ell^{2} \cdot \operatorname{genus}(C)+\frac{N_{0}}{\ell+1} \operatorname{deg} g_{k}^{*} \pi^{*} M .
\end{aligned}
$$

By combining this upper bound for the genus of $W_{k}$ with (4.3), we obtain that

$$
\operatorname{deg} g_{k}^{*}\left(\pi^{*} M\right) \leq \gamma \cdot \operatorname{genus}\left(W_{k}\right) \leq 3 \gamma(\operatorname{deg} \pi)^{2} \ell^{2} \cdot \operatorname{genus}(C)+\frac{N_{0}}{\ell+1} \operatorname{deg} g_{k}^{*} \pi^{*} M
$$

This implies that

$$
\operatorname{deg} g_{k}^{*}\left(\pi^{*} M\right) \leq 3 \gamma N_{0}(\operatorname{deg} \pi)^{2}(\ell+1)^{3} \cdot \operatorname{genus}(C) .
$$

In particular, we obtain that

$$
\operatorname{deg} f_{k}^{*} M=\frac{1}{\operatorname{deg} \pi} \operatorname{deg} g_{k}^{*} \pi^{*} M \leq 3 \gamma N_{0}(\operatorname{deg} \pi)(\ell+1)^{3} \cdot \operatorname{genus}(C) .
$$

Since the RHS of (4.8) is independent of $k$, it follows that $\left(f_{k}:\left(C, c_{0}\right) \rightarrow(X, x)\right)_{k=1}^{\infty}$ is an infinite sequence of pairwise distinct morphisms of bounded degree. In particular, it follows from Mori's bend-and-break (see [Deb01, Proposition 3.1]) that there is a rational curve in $X$ containing $x$. Since $x \notin \Delta$, this contradicts the fact that all rational curves of $X$ are contained in $\Delta$.

We conclude that $X$ is not geometrically-special.

Proposition 4.2 (The analogue of 3.1 in Yam10). Let $X$ be a smooth projective connected variety over $\mathbb{C}$, and let $K$ be a finite extension of $\mathbb{Q}_{p}$. Let $G$ be an almost simple algebraic group over $K$. Assume that there exists a p-unbounded representation $\rho: \pi_{1}(X) \rightarrow G(K)$ whose image is Zariski-dense in $G$. Then $X$ is not geometrically-special over $\mathbb{C}$.

Proof. By adapting the arguments in the first paragraph of Yamanoi's proof of [Yam10, Proposition 3.1], we will reduce to the case that $\rho$ is big, so that the result follows from Theorem 4.1 .

Assume, for a contradiction that $X$ is geometrically-special over $\mathbb{C}$. Note that, if $X^{\prime}$ is a smooth projective connected variety over $\mathbb{C}$ which is birational to $X$, then $X^{\prime}$ is geometrically-special (Lemma 2.8). Therefore, defining $H:=\operatorname{ker} \rho$, replacing $X$ by a smooth projective variety which is birational to $X$ if necessary, by the theory of Shafarevich maps (see [Kol93, p. 185] or [Cam94]), we have the $H$-Shafarevich morphism

$$
\operatorname{sh}_{X}^{H}: X \rightarrow \operatorname{Sh}^{H}(X)
$$

Let $F$ be a general fiber of $\operatorname{sh}_{X}^{H}$ and let $\pi_{1}(F)_{X}$ be the image of the natural morphism of groups $\pi_{1}(F) \rightarrow$ $\pi_{1}(X)$. Then, by definition of the $H$-Shafarevich map, the image

$$
\rho\left(\pi_{1}(F)_{X}\right) \subset G(K)
$$

is finite. It now follows from [uo99, Lemma 2.2.3] that there is a smooth projective connected variety $Y$ and a morphism $Y \rightarrow X$ which is the composition of a proper birational surjective morphism and a finite étale morphism such that, if $Y \rightarrow \Sigma$ denotes the Stein factorization of the composed morphism $Y \rightarrow X \rightarrow \operatorname{Sh}^{H}(X)$, then there exists a representation

$$
\rho_{\Sigma}: \pi_{1}(\Sigma) \rightarrow G(K)
$$

such that $\pi_{1}(Y) \rightarrow G(K)$ factors over $\rho_{\Sigma}$. By construction (see [Zuo99, Proposition 2.2.2]), the representation $\rho_{\Sigma}$ is big and has Zariski dense image in $G$. Let $\Sigma^{\prime}$ be a resolution of singularities of $\Sigma$. Note that the variety $Y$ is geometrically-special (Lemma 2.8 and Lemma 2.11). In particular, since $Y$ dominates 
$\Sigma$, it follows that $\Sigma$ and $\Sigma^{\prime}$ are geometrically-special (Lemma 2.8). Thus, $\Sigma^{\prime}$ is a smooth projective connected geometrically-special variety over $\mathbb{C}$ whose fundamental group has a big $p$-unbounded representation $\pi_{1}(X) \rightarrow G(K)$. This contradicts Theorem 4.1 .

4.2. Period maps. To prove that every linear quotient of the fundamental group of a geometrically-special variety is virtually abelian, we will use finiteness results for pointed maps to period domains. To state the necessary results, we recall the definition of a period map following Schmid [Sch73, Section 3] (see also [JLb, Section 4]).

Let $H$ be a finitely-generated free $\mathbb{Z}$-module, $k$ an integer, and $\left\{h^{p, k-p}\right\}$ a collection of non-negative integers with $h^{p, k-p}=h^{k-p, p}$ for all $p$,

$$
\sum_{p} h^{p, k-p}=\operatorname{rk}_{\mathbb{Z}} H
$$

Let $\hat{\mathscr{F}}$ be the flag variety parametrizing decreasing, exhaustive, separated filtrations of $H_{\mathbb{C}},\left(F^{\bullet}\right)$, with $\operatorname{dim} F^{p}=\sum_{i \geq p} h^{i, k-i}$. Let $\mathscr{F} \subset \hat{\mathscr{F}}$ be the analytic open subset of $\hat{\mathscr{F}}$ parametrizing those filtrations corresponding to $\mathbb{Z}$-Hodge structures of weight $k$, i.e. those filtrations with

$$
H_{\mathbb{C}}=F^{p}+\overline{F^{k-p+1}}
$$

for all $p$. Now suppose $q$ is a non-degenerate bilinear form on $H_{\mathbb{Q}}$, symmetric if $k$ is even and skew-symmetric if $k$ is odd. Let $D \subset \mathscr{F}$ be the locally closed analytic subset of $\mathscr{F}$ consisting of filtrations corresponding to polarized Hodge structures (relative to the polarization $q$ ), i.e. the set of filtrations $\left(F^{\bullet}\right)$ in $\mathscr{F}$ with

$$
q_{\mathbb{C}}\left(F^{p}, F^{k-p+1}\right)=0 \text { for all } p
$$

and

$$
q_{\mathbb{C}}(C v, \bar{v})>0
$$

for all nonzero $v \in H_{\mathbb{C}}$, where $C$ is the linear operator defined by $C(v)=i^{p-q} v$ for

$$
v \in H^{p, q}:=F^{p} \cap \overline{F^{q}} .
$$

Let $G=O(q)$ be the orthogonal group of $q$; it is a $\mathbb{Q}$-algebraic group. Write $G_{\mathbb{Z}}=G(\mathbb{Q}) \cap \mathrm{GL}(H)$, and let $\Gamma \subset G_{\mathbb{Z}}$ be a finite index subgroup.

If $X$ is smooth, then we say that a holomorphic map $X^{\text {an }} \rightarrow \Gamma \backslash D$ is a period map on $X$ if it is locally liftable and horizontal (i.e., satisfies Griffiths transversality). More generally, for $X$ a possibly singular variety, a holomorphic map $X^{\text {an }} \rightarrow \Gamma \backslash D$ is a period map on $X$ if there is a desingularization $\tilde{X} \rightarrow X$ such that the composed morphism $\tilde{X}^{\text {an }} \rightarrow X^{\text {an }} \rightarrow \Gamma \backslash D$ is a period map on $\tilde{X}$. We will make use of the following finiteness property for varieties with a quasi-finite period map which is essentially a reinterpretation of Deligne's finiteness result for monodromy representations.

Lemma 4.3 (Deligne, Deligne-Griffiths-Schmid). Let $X$ be a variety over $\mathbb{C}$. If $X$ admits a period map $X^{\text {an }} \rightarrow \Gamma \backslash D$ with finite fibres, then $X$ is geometrically hyperbolic over $\mathbb{C}$.

Proof. This follows from Deligne's finiteness theorem for monodromy representations [Del87] and the Theorem of the Fixed Part [Sch73, 7.24]. We refer the reader to [JLb, Theorem 5.1] for a detailed proof.

4.3. Period domains are far from being special. In [Yam10, Section 4], Yamanoi divides the task of proving his theorem into the rigid case and non-rigid case. To deal with the rigid case, he crucially uses that varieties which admit a non-constant period map are not Brody-special (i.e., do not admit a dense entire curve). The analogue for geometrically-special varieties of the statement he uses reads as follows.

Theorem 4.4. Let $X$ be a variety over $\mathbb{C}$. If $X$ admits a non-constant period map, then $X$ is not geometrically-special over $\mathbb{C}$.

Proof. Let $p: X^{\text {an }} \rightarrow \Gamma \backslash D$ be a non-constant period map. By Bakker-Brunebarbe-Tsimerman's resolution of Griffiths's conjecture [BBT], the analytic closure of the image of $p$ is the analytification $Y^{\text {an }}$ of a quasiprojective variety $Y$ over $\mathbb{C}$, and the induced dominant morphism $X^{\text {an }} \rightarrow Y^{\text {an }}$ is the analytification of a (non-constant dominant) morphism $f: X \rightarrow Y$. Note that $\operatorname{dim} Y>0$. Since $Y$ admits a quasifinite (even injective) period map, it is geometrically hyperbolic (Lemma 4.3). Since $X$ dominates the positive-dimensional geometrically hyperbolic variety $Y$, by Corollary 2.9, we conclude that $X$ is not geometrically-special over $\mathbb{C}$. 
4.4. The rigid case. We now deal with the case of a rigid representation $\rho: \pi_{1}(X) \rightarrow G(\mathbb{C})$.

Proposition 4.5 (The analogue of 4.1 in Yam10). Let $X$ be a smooth projective connected variety over $\mathbb{C}$, and let $G$ be an almost simple algebraic group over $\mathbb{C}$. Assume that there exists a rigid representation $\rho: \pi_{1}(X) \rightarrow G(\mathbb{C})$ whose image $\rho\left(\pi_{1}(X)\right)$ is Zariski-dense in $G$. Then $X$ is not geometrically-special over $\mathbb{C}$.

Proof. Since $\rho$ is rigid, it can be defined over some number field $K$. By abuse of notation, we let $\rho$ : $\pi_{1}(X) \rightarrow G(K)$ denote a model over $K$. For $p$ a finite place of $K$, we consider the representation

$$
\rho_{p}: \pi_{1}(X) \rightarrow G\left(K_{p}\right) .
$$

If there exists a finite place $p$ of $K$ such that $\rho_{p}$ is $p$-unbounded, then Proposition 4.2 implies that $X$ is not geometrically-special. Thus, we may and do assume that, for every finite place $p$ of $K$, the representation $\rho_{p}$ is $p$-bounded. In this case, as is explained by Yamanoi in the proof of [Yam10, 4.1], the subgroup $\rho^{-1}\left(G\left(\mathcal{O}_{K}\right)\right)$ is of finite index in $\pi_{1}(X)$. Therefore, there is a finite étale cover $Y \rightarrow X$ such that $\rho\left(\pi_{1}(Y)\right) \subset$ $G\left(\mathcal{O}_{K}\right)$. Then, as $\left.\rho\right|_{\pi_{1}(Y)}: \pi_{1}(Y) \rightarrow G\left(\mathcal{O}_{K}\right)$ is a rigid representation, by a result of Simpson (see Sim92, p. 58]), the variety $Y$ admits a period map, say $p: Y^{\text {an }} \rightarrow \Gamma \backslash D$, whose rational monodromy representation contains $\left.\rho\right|_{\pi_{1}(Y)}$ as a direct factor. Since $\rho$ has Zariski dense image in $G$, it follows that the period map $p$ is non-constant. We conclude that $Y$ is not geometrically-special from Theorem 4.4 In particular, as $Y \rightarrow X$ is finite étale, the variety $X$ is not geometrically-special (Lemma 2.11). This concludes the proof.

4.5. The general case. To deal with the non-rigid case, we begin with the following result proven by Yamanoi which we state in more generality than necessary.

Proposition 4.6. Let $X$ be a variety over $\mathbb{C}$, and let $G$ be an almost simple algebraic group over $\mathbb{C}$. Assume that there exists a non-rigid representation $\rho: \pi_{1}(X) \rightarrow G(\mathbb{C})$ whose image $\rho\left(\pi_{1}(X)\right)$ is Zariskidense in $G$. Then, for every prime number $p$, there exists a finite extension $K / \mathbb{Q}_{p}$ and a $p$-unbounded representation $\widetilde{\rho}: \pi_{1}(X) \rightarrow G(K)$ whose image is Zariski-dense in $G$.

Proof. This is shown in the proof of [Yam10, Lemma 4.1]. (The argument only uses properties of character varieties.)

Proposition 4.6 allows us to deal with non-rigid representations by appealing to our result for $p$ unbounded representations.

Corollary 4.7 (The analogue of 2.1 in Yam10]). Let $X$ be a smooth projective connected variety over $\mathbb{C}$, and let $G$ be an almost simple algebraic group over $\mathbb{C}$. Assume that there exists a representation $\rho: \pi_{1}(X) \rightarrow G(\mathbb{C})$ whose image $\rho\left(\pi_{1}(X)\right)$ is Zariski-dense in $G$. Then, the variety $X$ is not geometricallyspecial over $\mathbb{C}$.

Proof. If $\rho$ is rigid, then the result follows from Proposition 4.5, so we may assume that $\rho$ is non-rigid. Let $p$ be a prime number. Since $\rho$ is a non-rigid representation whose image is Zariski-dense in $G$, it follows from Proposition 4.6 that there is a $p$-adic field $K$ and a $p$-unbounded representation $\widetilde{\rho}: \pi_{1}(X) \rightarrow G(K)$ whose image is Zariski-dense in $G$. Then, by Proposition 4.2, the variety $X$ is not geometrically-special over $\mathbb{C}$.

4.6. Linear quotients of fundamental groups. Corollary 4.7 says that the fundamental group $\pi_{1}(X)$ of a smooth projective connected geometrically-special variety $X$ over $\mathbb{C}$ does not admit a Zariski dense representation into an almost simple algebraic group. We now combine this result with the structure result for Albanese maps of such varieties to show that linear quotients of $\pi_{1}(X)$ are virtually abelian.

Proof of Theorem 1.15, Assume that $X$ is geometrically-special over $\mathbb{C}$, and let $\rho: \pi_{1}(X) \rightarrow \mathrm{GL}_{n}(\mathbb{C})$ be a representation. We follow Yamanoi's "Proposition 2.1 implies Theorem 1.1" in Yam10. Thus, let $H$ be the Zariski closure of the image of $\rho$ in $\mathrm{GL}_{n}(\mathbb{C})$. Since every finite étale cover of $X$ is geometrically-special (Lemma 2.11), replacing $X$ by a finite étale cover, we may and do assume that $H$ is connected. Then, as $H / R(H)$ is an almost direct product of almost simple algebraic groups, it follows from Corollary 4.7 that $H$ equals its radical $R(H)$. Therefore, the image $\rho\left(\pi_{1}(X)\right)$ is a solvable group.

We now apply a theorem of Campana [Cam01, Theoreme 2.9]. First, note that the Albanese map of every finite étale covering $X^{\prime}$ of $X$ is surjective (Corollary 3.7). Therefore, it follows from [Cam01, Theoreme 2.9] 
that there exists a finite étale morphism $Y \rightarrow X$ such that $\pi_{1}(Y) \rightarrow H$ factors over the induced group homomorphism $\pi_{1}(Y) \rightarrow \pi_{1}(\operatorname{Alb}(Y))$. Since $\pi_{1}(\operatorname{Alb}(Y))$ is abelian, this implies that $\rho\left(\pi_{1}(Y)\right)$ is abelian. We conclude that $\rho\left(\pi_{1}(X)\right)$ is virtually abelian.

Proof of Theorem 1.16. We follow and adapt Yamanoi's proof of [Yam10, Corollary 1.3].

Let $X$ be a geometrically-special smooth projective variety over $\mathbb{C}$ such that there is a big representation $\rho: \pi_{1}(X) \rightarrow \mathrm{GL}_{n}(\mathbb{C})$. Then, by Theorem 1.15, the image of $\rho$ is virtually abelian. Thus, as every finite étale cover of $X$ is geometrically-special (Lemma 2.11), replacing $X$ by a suitable finite étale cover if necessary, we may and do assume that the image of $\pi_{1}(X)$ is a free abelian group. Since the image of $\rho$ is a torsion-free abelian group and $\pi_{1}(\operatorname{Alb}(X))$ is the torsion-free abelianization of $\pi_{1}(X)$, it follows that $\rho$ factors over the homomorphism $\left.\pi_{(} X\right) \rightarrow \pi_{1}(\operatorname{Alb}(X))$ induced by the Albanese map $a_{X}: X \rightarrow \operatorname{Alb}(X)$. To conclude the proof, we now show that the Albanese map $a_{X}: X \rightarrow \operatorname{Alb}(X)$ is birational.

Since $X$ is geometrically-special, the Albanese map $a_{X}$ is surjective with connected fibers (Corollary 3.11). Let $F$ be a general fiber of $a_{X}$. Then $\rho\left(\operatorname{Im}\left(\pi_{1}(F) \rightarrow \pi_{1}(X)\right)\right)$ is trivial. In particular, since $\rho$ is big, the fiber $F$ is a point. From this it follows that $a_{X}$ is birational, as required.

\section{REFERENCES}

[BBT] B. Bakker, Y. Brunebarbe, and J. Tsimerman. o-minimal GAGA and a conjecture of Griffiths. arXiv:1811.12230.

[BG06] E. Bombieri and W. Gubler. Heights in Diophantine geometry, volume 4 of New Mathematical Monographs. Cambridge University Press, Cambridge, 2006.

[BJK] R. van Bommel, A. Javanpeykar, and L. Kamenova. Boundedness in families with applications to arithmetic hyperbolicity. arXiv:1907.11225.

[Bog77] F. A. Bogomolov. Families of curves on a surface of general type. Dokl. Akad. Nauk SSSR, 236(5):1041-1044, 1977.

[Cam94] F. Campana. Remarques sur le revêtement universel des variétés kählériennes compactes. Bull. Soc. Math. France, 122(2):255-284, 1994.

[Cam01] F. Campana. Ensembles de Green-Lazarsfeld et quotients résolubles des groupes de Kähler. J. Algebraic Geom., 10(4):599-622, 2001.

[Cam04] F. Campana. Orbifolds, special varieties and classification theory. Ann. Inst. Fourier (Grenoble), 54(3):499-630, 2004.

[Cam11] F. Campana. Orbifoldes géométriques spéciales et classification biméromorphe des variétés kählériennes compactes. Journal de l'Institut de Mathématiques de Jussieu, 10(4):809-934, 2011.

[CC16] F. Campana and B. Claudon. Quelques propriétés de stabilité des variétés spéciales. Math. Z., 283(1-2):581-599, 2016.

[CCE15] F. Campana, B. Claudon, and P. Eyssidieux. Représentations linéaires des groupes kählériens: factorisations et conjecture de Shafarevich linéaire. Compos. Math., 151(2):351-376, 2015.

$\left[\mathrm{CDJ}^{+}\right]$P. Corvaja, J.L. Demeio, A. Javanpeykar, D. Lombardo, and U. Zannier. On the distribution of rational points on ramified covers of abelian varieties. arXiv:2011.12840.

[Deb01] O. Debarre. Higher-dimensional algebraic geometry. Universitext. Springer-Verlag, New York, 2001.

[Del87] P. Deligne. Un théorème de finitude pour la monodromie. In Discrete groups in geometry and analysis (New Haven, Conn., 1984), volume 67 of Progr. Math., pages 1-19. Birkhäuser Boston, Boston, MA, 1987.

[Dem97] J.-P. Demailly. Variétés projectives hyperboliques et équations différentielles algébriques. In Journée en l'Honneur de Henri Cartan, volume 1997 of SMF Journ. Annu., pages 3-17. Soc. Math. France, Paris, 1997.

[Fal94] G. Faltings. The general case of S. Lang's conjecture. In Barsotti Symposium in Algebraic Geometry (Abano Terme, 1991), volume 15 of Perspect. Math., pages 175-182. Academic Press, San Diego, CA, 1994.

[FJ74] G. Frey and M. Jarden. Approximation theory and the rank of abelian varieties over large algebraic fields. Proc. London Math. Soc. (3), 28:112-128, 1974.

[Gro72] A. Grothendieck. Groupes de monodromie en géométrie algébrique (SGA 7 I). Lecture Notes in Mathematics, Vol. 288. Springer-Verlag, Berlin-New York, 1972. Séminaire de Géométrie Algébrique du Bois-Marie 1967-1969.

[Has03] B. Hassett. Potential density of rational points on algebraic varieties. In Higher dimensional varieties and rational points (Budapest, 2001), volume 12 of Bolyai Soc. Math. Stud., pages 223-282. Springer, Berlin, 2003.

[Jav] A. Javanpeykar. The Lang-Vojta conjectures on projective pseudo-hyperbolic varieties. Chapter 3 in Arithmetic Geometry of Logarithmic Pairs and Hyperbolicity of Moduli Spaces. CRM Short Courses Springer.

[Jav18] A. Javanpeykar. Arithmetic hyperbolicity: automorphisms and persistence. Math. Ann., to appear. arXiv:1809.06818, 2018.

[JK20] A. Javanpeykar and L. Kamenova. Demailly's notion of algebraic hyperbolicity: geometricity, boundedness, moduli of maps. Math. Z., 296(3-4):1645-1672, 2020.

[JLa] A. Javanpeykar and A. Levin. Urata's theorem in the logarithmic case and applications to integral points. arXiv:2002.11709.

[JLb] A. Javanpeykar and D. Litt. Integral points on algebraic subvarieties of period domains: from number fields to finitely generated fields. arXiv:1907.13536. 
[JSZ] A. Javanpeykar, R. Sun, and K. Zuo. The Shafarevich conjecture revisited: Finiteness of pointed families of polarized varieties. arXiv:2005.05933.

[JX] A. Javanpeykar and J. Xie. Finiteness properties of pseudo-hyperbolic varieties. IMRN, to appear. arXiv:1909.12187. [Kaw80] Y. Kawamata. On Bloch's conjecture. Invent. Math., 57(1):97-100, 1980.

[Kaw81] Y. Kawamata. Characterization of abelian varieties. Compositio Math., 43(2):253-276, 1981.

[Kob98] S. Kobayashi. Hyperbolic complex spaces, volume 318 of Grundlehren der Mathematischen Wissenschaften [Fundamental Principles of Mathematical Sciences]. Springer-Verlag, Berlin, 1998.

[Kol93] J. Kollár. Shafarevich maps and plurigenera of algebraic varieties. Invent. Math., 113(1):177-215, 1993.

[Lan86] S. Lang. Hyperbolic and Diophantine analysis. Bull. Amer. Math. Soc. (N.S.), 14(2):159-205, 1986.

[LW12] S. S. Y. Lu and J. Winkelmann. Quasiprojective varieties admitting Zariski dense entire holomorphic curves. Forum Math., 24(2):399-418, 2012.

[Moc12] S. Mochizuki. Topics in absolute anabelian geometry I: generalities. J. Math. Sci. Univ. Tokyo, 19(2):139-242, 2012.

[Mum08] D. Mumford. Abelian varieties, volume 5 of Tata Institute of Fundamental Research Studies in Mathematics. Published for the Tata Institute of Fundamental Research, Bombay; by Hindustan Book Agency, New Delhi, 2008.

[NWY07] J. Noguchi, J. Winkelmann, and K. Yamanoi. Degeneracy of holomorphic curves into algebraic varieties. J. Math. Pures Appl. (9), 88(3):293-306, 2007.

[Oes85] J. Oesterlé. Courbes sur une variété abélienne (d'après M. Raynaud). Astérisque, (121-122):213-224, 1985. Seminar Bourbaki, Vol. 1983/84

[Ray83] M. Raynaud. Courbes sur une variété abélienne et points de torsion. Invent. Math., 71(1):207-233, 1983.

[Rou10] E. Rousseau. Hyperbolicity of geometric orbifolds. Trans. Amer. Math. Soc., 362(7):3799-3826, 2010.

[Rou12] E. Rousseau. Degeneracy of holomorphic maps via orbifolds. Bull. Soc. Math. France, 140(4):459-484 (2013), 2012.

[Sch73] W. Schmid. Variation of Hodge structure: the singularities of the period mapping. Invent. Math., 22:211-319, 1973.

[Sim92] C. T. Simpson. Higgs bundles and local systems. Inst. Hautes Études Sci. Publ. Math., (75):5-95, 1992.

[Sta15] The Stacks Project Authors. Stacks Project. http://stacks.math.columbia.edu, 2015.

[Uen75] K. Ueno. Classification theory of algebraic varieties and compact complex spaces. Lecture Notes in Mathematics, Vol. 439. Springer-Verlag, Berlin-New York, 1975. Notes written in collaboration with P. Cherenack.

[Yam10] K. Yamanoi. On fundamental groups of algebraic varieties and value distribution theory. Ann. Inst. Fourier (Grenoble), 60(2):551-563, 2010.

[Yam15] K. Yamanoi. Holomorphic curves in algebraic varieties of maximal Albanese dimension. Internat. J. Math., 26(6):1541006, 45, 2015

[Zuo96] K. Zuo. Kodaira dimension and Chern hyperbolicity of the Shafarevich maps for representations of $\pi_{1}$ of compact Kähler manifolds. J. Reine Angew. Math., 472:139-156, 1996.

[Zuo99] K. Zuo. Representations of fundamental groups of algebraic varieties, volume 1708 of Lecture Notes in Mathematics. Springer-Verlag, Berlin, 1999.

Ariyan Javanpeykar, Institut für Mathematik, Johannes Gutenberg-Universität Mainz, Staudingerweg 9 , 55099 Mainz, Germany.

Email address: peykar@uni-mainz.de

Erwan Rousseau, institut Universitaire de France \& Aix Marseille Univ, CNRS, Centrale Marseille, i2m, Marseille, France

Email address: erwan.rousseau@univ-amu.fr 